\title{
Circ-SIRT1 inhibits cardiac hypertrophy via activating SIRT1 to promote autophagy
}

\author{
Weichen Wang ${ }^{1}$, Longlong Wang ${ }^{1}$, Mengyue $\mathrm{Yang}^{1}$, Chunwei $\mathrm{Wu}^{1}$, Rui Lan ${ }^{1}$, Weiwei Wang ${ }^{1}$ and Yuze $\mathrm{Li}^{1 凶}$ \\ (c) The Author(s) 2021
}

Mounting studies have substantiated that abrogating autophagy contributes to cardiac hypertrophy (CH). Sirtuin 1 (SIRT1) has been reported to support autophagy and inhibit $\mathrm{CH}$. However, the upstream regulation mechanism behind the regulation of SIRT1 level in $\mathrm{CH}$ remains unclear. Circular RNAs (circRNAs) are vital modulators in diverse human diseases including $\mathrm{CH}$. This study intended to investigate the regulatory mechanism of circRNA on SIRT1 expression in $\mathrm{CH}$. CH model was established by angiotensin II (Ang II) fusion or transverse aortic constriction (TAC) surgery and Ang II treatment on hiPSC-CMs and H9c2 cells in vitro. Our results showed that circ-SIRT1 (hsa_circ_0093884) expression was downregulated in Ang II-treated hiPSC-CMs, and confirmed that its conserved mouse homolog circ-Sirt1 (mmu_circ_0002354) was expressed at low levels in Ang II-treated H9c2 cells and TAC-induced mice model. Functionally, circ-SIRT1/circ-Sirt1 attenuated Ang II-induced CH and induced autophagy in hiPSC-CMs and H9c2 cardiomyocytes. Mechanistically, circ-SIRT1 could upregulate its host gene SIRT1 at the post-transcriptional level by sponging miR$3681-3 p / m i R-5195-3 p$ and stabilized SIRT1 protein at the post-translational level by recruiting USP22 to induce deubiquitination on SIRT1 protein. Further, SIRT1 knockdown could rescue the effect of circ-SIRT1 upregulation on Ang II-induced CH and autophagy in vitro and in vivo. In conclusion, we first uncovered that circ-SIRT1 restrains CH via activating SIRT1 to promote autophagy, indicating circ-SIRT1 as a promising target to alleviate $\mathrm{CH}$.

Cell Death and Disease (2021)12:1069; https://doi.org/10.1038/s41419-021-04059-y

\section{INTRODUCTION}

Cardiac hypertrophy $(\mathrm{CH})$, featuring enlarged cardiomyocytes as well as heart mass, is generally depicted as a significant compensatory mechanism of the heart in response to diverse physiological and pathological overloads, and it helps sustain cardiac function in its original stage [1, 2]. Unfortunately, continuous $\mathrm{CH}$ is related to dysfunction and cardiac remodeling, which ultimately leads to lower compliance, a higher risk of congestive heart failure, and sudden death, so $\mathrm{CH}$ has a high rate of death all over the world [3-5]. Pathological $\mathrm{CH}$ is a common risk factor for heart failure and multiple factors contributed to $\mathrm{CH}$, including aging and neurohumoral activation (e.g., Ang II) [6]. Antiaging approaches, such as the restriction of caloric, can benefit cardiac functions in rodents, monkeys as well as human beings [7-9]. Although researchers are working effortlessly to study $\mathrm{CH}$ pathology and have uncovered that multiple pathological stimuli (i.e., specific peptide hormones and growth factors) are implicated in $\mathrm{CH}$ regulation, it remains difficult to fully explain the complicated molecular mechanisms in $\mathrm{CH}$ [10]. Thus, to improve the quality of $\mathrm{CH}$ treatment and prevent ultimate heart failure, it is of considerable significance to identify potent therapeutic targets that may modulate $\mathrm{CH}$.

Over the past decade, RNA molecules have been revealed to participate in the regulation of various diseases, including $\mathrm{CH}$ $[11,12]$. Commonly, mRNAs are involved in the biological process of cancer progression by directly coding functional proteins [13].
SIRT1, a kind of mRNA with protein-coding potential, has been reported to promote autophagy by de-acetylation of multiple autophagy-related genes such as Beclin1 and ATG9A as reported by previous studies $[14,15]$. Interestingly, it is acknowledged that promoting autophagy can attenuate $\mathrm{CH}[16,17]$, and accumulating evidence supports that SIRT1 has an inhibitive effect on $\mathrm{CH}$ [18-20]. However, the upstream mechanism of regulating SIRT1 expression in $\mathrm{CH}$ remains largely unknown.

Most mammalian genomes can be transcribed into noncoding RNAs (ncRNAs) [21]. Traditionally, ncRNAs are predominantly categorized into two subtypes depending on size: long noncoding RNAs (IncRNAs) with over 200 nucleotides in length, and small noncoding RNAs (microRNAs [miRNAs] included) featured with 20-23 nucleotide small RNAs [22]. Emerging as a new star in molecular researches, circular RNAs (circRNAs) are a group of ncRNAs characterized by a closed continuous loop structure, in which the $3^{\prime}$ - and $5^{\prime}$-ends are joined together by back splicing $[23,24]$. In recent years, circRNAs have also been recognized as competing endogenous RNAs (ceRNAs) which can sponge miRNAs by complementary base pairing. The regulation of circRNAs on SIRT1 has been revealed in different types of human tumors and diseases. For example, hsa_circ_0001946 propels cell growth in lung adenocarcinoma via the serving as a ceRNA regulating miR135a-5p/SIRT1 axis [25]. Moreover, hsa_circ_0076248 enhances the tumorigenesis of glioma by acting as a miRNA sponge for miR-181a to regulate SIRT1 expression [26]. Circ-SIRT1 (hsa_circ_0093887),

${ }^{1}$ Department of Cardiology, the First Hospital of China Medical University, 110001 Shenyang, Liaoning, People's Republic of China. ${ }^{凶}$ email: jrhs@163.com Edited by I Amelio

Received: 19 January 2020 Revised: 20 July 2021 Accepted: 26 July 2021

Published online: 10 November 2021 
derived from the circularization of exon 2 to exon-7 of the SIRT1 gene, has been reported to promote SIRT1 expression by sponging miR-132/212 in vascular smooth muscle cells [27]. Through preliminary experiments, we discovered that circ-SIRT1 was downregulated by Ang II in cardiomyocytes. However, the relation between circ-SIRT1 and SIRT1 in CH has never been studied.

Thus, this study aimed to uncover the regulation of circSIRT1 on SIRT1 and the function of circ-SIRT1/SIRT1 axis in $\mathrm{CH}$. We established in vitro and in vivo $\mathrm{CH}$ models and applied a string of functional assays and molecular mechanism experiments to probe into the potential upstream regulation of SIRT1 including circ-SIRT1 (hsa_circ_0093884) and its conserved mouse homolog circ-Sirt1 (mmu_circ_0002354) in CH, attempting to identify circ-SIRT1 as a novel target for the improvement of $\mathrm{CH}$ treatment.

\section{MATERIALS AND METHODS \\ Cell culture and treatment}

Human-induced pluripotent stem cell-derived cardiomyocytes (hiPSC-CMs) were available from Beijing Cellapy Biotechnology Co., Ltd. (Beijing, China), and the rat heart-derived $\mathrm{H} 9 \mathrm{C} 2$ cardiomyocytes were available from the Chinese Academy of Sciences (Shanghai, China). Both cardiomyocytes were grown in DMEM (Dulbecco's Modified Eagle Medium; Invitrogen, Carlsbad, CA, USA) under $5 \% \mathrm{CO}_{2}$ at $37^{\circ} \mathrm{C}$. In all, 10\% FBS (fetal bovine serum; HyClone, South Logan, UT, USA) and $1 \%$ antibiotics acted as the medium supplements. The in vitro $\mathrm{CH}$ cell model was established in both cardiomyocytes with the $24 \mathrm{~h}$ of treatment of $150 \mathrm{nM}$ of Ang II (SigmaAldrich, St. Louis, MO, USA) or $50 \mu \mathrm{M}$ of isoproterenol (ISO; GuideChem, China) for $24 \mathrm{~h}$. In total, $3 \mathrm{U} / \mu \mathrm{g}$ of RNase $\mathrm{R}$ was procured from Epicentre Technologies (Madison, WI, USA). The cycloheximide $(\mathrm{CHX} ; 40 \mu \mathrm{g} / \mathrm{mL}$ ) was also acquired from Sigma-Aldrich (St. Louis, MO, USA). The 3-methyladenine (3-MA; an autophagy inhibitor) was obtained from Abcam (Cambridge, MA, USA).

\section{Plasmid transfection}

The designed shRNAs and NC-shRNAs (Genepharma Company, Shanghai, China) were applied for silencing circ-SIRT1 (or circ-Sirt1), USP22, and SIRT1. Besides, miR-3681-3p mimics/inhibitor and NC mimics/inhibitor as well as the pcDNA3.1(+)/circ-SIRT1 (circ-SIRT1-oe) or pcDNA3.1(+)/circ-Sirt1 (circSirt1-oe) or empty pcDNA3.1(+) CircRNA Mini Vector (pcDNA3.1 $(+))$, were all procured from Genepharma Company. All plasmids were transfected into hiPSC-CMs and $\mathrm{H} 9 \mathrm{c} 2$ cardiomyocytes for $48 \mathrm{~h}$ employing the Lipofectamine 2000 (Invitrogen, Carlsbad, CA, USA). Three independent bio-repeats were conducted in the experiment and each bio-repeat contains three technical replicates.

\section{Animal study}

To establish the $\mathrm{CH}$ mouse model, male C57BL/6 mice $(n=12)$, aging 8week-old and weighing 20-25 g, were commercially acquired from Beijing Vital River Laboratory Animal Technology Co., Ltd. (Beijing, China). The animal-related protocol was approved by the Animal Care and Use Committee of the First Hospital of China Medical University. The CH mouse model was established via Transverse Aortic Constriction (TAC) surgery $(N=6)$. After anesthesia with intraperitoneal ketamine and xylazine, the transverse thoracic aorta was dissected from the mouse model. Mice were then placed on a ventilator for 1 week to recover the mouse model. The pressure gradient in the TAC group was examined by echocardiography. Mice of the sham group $(N=6)$ were subjected to the same experimental procedures as the TAC group, except the aorta seam. Besides, we constructed Ang II-treated in vivo model by chronic subcutaneous infusion of Ang II into mice $(N=6)$, saline as control $(N=6)$. To further analyze the effects of circ-Sirt1 on Ang II-treated in vivo model, adenovirus (Biocobio, Tianjin, China) of circ-Sirt1 or pcDNA3.1 $(+)$ was injected into the mice left ventricle myocardium 7 days before TAC surgery.
\end{abstract}

\section{Enzyme-linked immunosorbent assay (ELISA)}

Serum was diluted using PBS, cardiac tissues with the same weight in the sham and TAC groups were prepared via homogenization in PBS. Ang II concentrations in serum were measured via ELISA kit (R\&D Systems, Inc., Minneapolis, MN, USA) following the supplier's protocols. All samples were analyzed via two bio-repeats and two technical replicates.

\section{Immunofluorescence staining for cell surface area measurement}

Mice heart cells, cardiomyocytes (hiPSC-CMs and H9c2) were seeded in 96-well plates and then washed twice with phosphate-buffered saline (PBS). Subsequently, cardiomyocytes were fixed by $4 \%$ formaldehyde for $20 \mathrm{~min}$, permeated by $0.1 \%$ Triton X-100, and then incubated with aactinin antibody (Cat \#: 6487; 1/100, Cell Signaling Technology, Danvers, MA, USA) at $4{ }^{\circ} \mathrm{C}$ overnight. The secondary antibody (Cat \#: 4410; $1 /$ 1000, Cell Signaling Technology) was used to culture cells at room temperature for $1 \mathrm{~h}$. After DAPI staining, immunofluorescence was observed under a fluorescence microscope (Olympus Corp., Tokyo, Japan) for the estimation of cell surface area. In all, 50 independent cells were analyzed per sample. The researchers performing the analysis were blinded to the experimental groups. Three independent biorepeats were conducted in the experiment and each bio-repeat contains three technical replicates. The results were analyzed with Image-Pro Plus Data Analysis software.

\section{RNA extraction and RT-qPCR}

TRIZOL reagent (Invitrogen, Carlsbad, CA, USA) was used for the extraction of total RNA from cardiomyocytes. Then, the reverse transcription was accomplished as per the supplier's instructions (Invitrogen, Carlsbad, CA, USA). To examine gene expression, RT-qPCR was carried out on ABI 7500 Fast Real-Time PCR System (Applied Biosystems, Foster City, CA, USA). Data were processed by $2^{-\Delta \Delta \mathrm{Ct}}$ method and standardized to $\mathrm{U} 6$ or GAPDH (glyceraldehyde-3-phosphate dehydrogenase). Three independent biorepeats were conducted in the experiment and each bio-repeat contains three technical replicates.

\section{Western blot}

The cultured cardiomyocytes were lysed in RIPA lysis buffer, then separated on 12\% SDS-PAGE (sodium dodecyl sulfate-polyacrylamide gel electrophoresis) and transferred to PVDF membranes. After being blocked in 5\% skim milk, primary antibodies against GAPDH (Cat. \#: ab8245; 1/5000, Abcam, Cambridge, UK; control) and ANF (atrial natriuretic factor; Cat. \#: ab225844, 1/1000, Abcam), BNP (brain natriuretic peptide; Cat. \#: ab92500, 1/10,000 Abcam), $\beta$-MHC ( $\beta$-myosin heavy chain; Cat. \#: ab170867, 1/3000, Abcam), p62 (Cat. \#: ab109012, 1/ 30,000, Abcam), LAMP1 (lysosomal associated membrane protein 1; Cat. \#: ab108597, 1/5000, Abcam), SIRT1 (Cat. \#: ab32441, 1/20,000, Abcam) and USP22 (ubiquitin-specific peptidase 22; Cat. \#: ab195289, 1/2000, Abcam) were used. Anti-LC3 (light chain 3) antibody (Cat. \#: 14600-1-AP, $1 / 1500$, Proteintech, Rosemont, IL, USA) was procured from SigmaAldrich (St. Louis., MO, USA). To be noted, there are two forms of LC3 in various cells, called LC3-I and - II; LC3-I is cytosolic, whereas LC3-II is membrane-bound [28]. After overnight incubation, the HRP-tagged secondary antibodies (Cat. \#: ab6728; 1/5000, Abcam) were added for $2 \mathrm{~h}$ at room temperature. All protein bands were examined by the $\mathrm{ECL}$ detection system (Pierce, Rockford, IL, USA). Western blot results of proteins of interest in the cardiomyocytes were quantified with the help of ImageJ software and statistical analysis was conducted via SPSS version 13.0. Three independent bio-repeats were conducted in the experiment and each bio-repeat contains three technical replicates.

\section{Subcellular fractionation}

In total, $1 \times 10^{6}$ hiPSC-CMs and $\mathrm{H} 9 \mathrm{c} 2$ cardiomyocytes were washed in pre-cooled PBS twice for $5 \mathrm{~min}$ of centrifugation at $500 \times \mathrm{g}$ at $4^{\circ} \mathrm{C}$. Cytoplasmic and nuclear fractions were individually extracted using PARIS $^{\text {TM }}$ Kit (Invitrogen, Carlsbad, CA, USA) following the manufacturer's direction. Expression levels of circ-SIRT1 (or circ-Sirt1) in both fractions were assayed by RT-qPCR, with GAPDH and U6 as controls for cell cytoplasm and cell nucleus, respectively. Three independent bio-repeats were conducted in the experiment and each bio-repeat contains three technical replicates.

\section{Fluorescence in situ hybridization (FISH)}

$\mathrm{H} 9 \mathrm{C} 2$ and hiPSC-CMs cardiomyocytes were fixed and processed with pepsin, then dehydrated in ethanol. Cells were incubated in hybridization buffer with $40 \mathrm{nM}$ of circ-SIRT1 (or circ-Sirt1)-FISH probe (Ribobio, Guangzhou, China). Following nuclear detection with DAPI solution, cells were detected visually by fluorescence microscope (Olympus Corp., Tokyo, Japan). Three independent bio-repeats were conducted in the experiment and each bio-repeat contains three technical replicates. 


\section{GFP-mRFP-LC3 adenoviral transfection}

$\mathrm{H} 9 \mathrm{c} 2$ and hiPSC-CMs cardiomyocytes were planted on the glass-bottomed cell culture dishes and then infected with the adenoviral vectors GFPmRFP-LC3 (Ad-GFP-mRFP-LC3; HanBio Technology, Shanghai, China) expressing the GFP and mRFP fluorescent proteins for marking and tracking LC3 to monitor autophagic influx. Then, the culture medium was changed with the fresh medium, and cells were observed by confocal laser scanning microscope (Zeiss, Dublin, CA, USA) after $24 \mathrm{~h}$ to analyze autophagy flux and the number of green, red (autolysosomes), and yellow dots (autophagosomes), which were quantified with the application of Image Plus Pro Software. More than ten cells in three independent biorepeats were analyzed randomly in the experiment and each bio-repeat contains three technical replicates.

\section{RNA pull-down assays}

For RNA-RNA pull-down assays, the cardiomyocytes were subjected to icecold PBS and lysis buffer and then incubated with circ-SIRT1 biotin probes (TCTGAAGAGCTCTGTGACCC-biotin) or NC-biotin probes (AGACTTCTCGACTGTGACCC-biotin) at room temperature for $2 \mathrm{~h}$. After the addition of streptavidin magnetic beads (Invitrogen, Carlsbad, CA, USA), the biotincoupled circ-SIRT1 complex was incubated for another $4 \mathrm{~h}$ at $4{ }^{\circ} \mathrm{C}$, followed by washing with RIP washing buffer. Subsequently, the binding miRNAs were extracted from the pulldowns using TRlzol and detected using qRTPCR. Enrichment values of miRNA (miR-3681-3p, miR-4766-5p, miR-889-3p, or miR-5195-3p) were compared to NC-biotin probe control.

To verify the interaction between miR-3681-3p/miR-5195-3p and circSIRT1 and SIRT1, biotinylated miR-3681-3p or miR-5195-3p (Bio-miR-3681$3 p:$ acacagugcuucauccacuacu-biotin; Bio-miR-5195-3p: auccaguucucugagggggcu-biotin) was incubated with streptavidin magnetic beads. Then, circ-SIRT1 and SIRT1 in the pulldowns were isolated using TRIzol and subjected to qRT-PCR analysis. Enrichment values of circ-SIRT1/SIRT1 were relative to Bio-NC control, and the data were mean \pm standard variation (SD) of three replicates. For RNA-protein pull-down assays, Pierce Magnetic RNA-Protein Pull-Down Kit was acquired from Thermo Fisher (Waltham, MA) for RNA-protein pull-down assay in cardiomyocytes. The protein extracts were prepared and set as three groups, including Input, circ-SIRT1 biotin probe, and circ-SIRT1 no-biotin probe. The protein extracts were mixed with the biotin-tagged circ-SIRT1 probes. The magnetic beads were added for $1 \mathrm{~h}$. Following RNA-protein pull-down assay, the circ-SIRT1 interacting proteins were subjected to SDS-PAGE and silver staining, followed by mass spectrometry analysis (CapitalBio Technology, Beijing, China) of the specific band. Besides, the enriched USP22 was detected by western blot. Three independent bio-repeats were conducted in the experiment and each bio-repeat contains three technical replicates.

\section{RNA immunoprecipitation (RIP)}

Magna RIP ${ }^{\mathrm{TM}}$ RNA-Binding Protein Immunoprecipitation Kit was available for RIP assay in $1 \times 10^{7}$ cardiomyocytes as required by the supplier (Millipore, Bedford, MA, USA). Cell lysates were incubated with RIP buffer adding the magnetic beads and specific antibodies to human Ago2 (Cat. \#: 2897; 1/50, Cell Signaling Technology) and USP22 (Cat. \#: ab195289; 1/40, Abcam). Normal mouse IgG antibody (Cat. \#: 3420; 1/20, Cell Signaling Technology) was used in the control group. Three independent bio-repeats were conducted in the experiment and each bio-repeat contains three technical replicates.

\section{Co-immunoprecipitation (ColP)}

The cardiomyocytes were harvested by centrifugation at $400 \times g$ for $3 \mathrm{~min}$. The processed cardiomyocytes were re-suspended in IP lysis buffer and subsequently left on ice for $15 \mathrm{~min}$. After that, the cardiomyocytes were sonicated for $2 \times 10 \mathrm{~s}$ and placed directly on ice, followed by centrifugation at $10,000 \times g$ for $10 \mathrm{~min}$ at $4{ }^{\circ} \mathrm{C}$. Afterward, the supernatant was transferred to a fresh cold Eppendorf tube. Bradford assay was applied to determine the protein concentration. The lysate was incubated with the specific primary antibodies against myc (Cat. \#: 2276; 1/1000, Cell Signaling Technology), Flag (Cat. \#: 14793; 1/50, Cell Signaling Technology), SIRT1 (Cat. \#: 8469; 1/100, Cell Signaling Technology), USP22 (Cat. \#: ab195289,

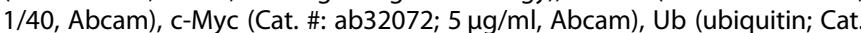
\#: 62802; 1/100, Cell Signaling Technology), or IgG antibody (Cat. \#: 3420; $1 / 20$, Cell Signaling Technology) plus A/G sepharose beads or $A / G$ sepharose beads only on a rotator overnight at $4{ }^{\circ} \mathrm{C}$. Then, cell samples were washed in IP lysis buffer and boiled in 4x SDS-loading buffer for western blot analysis. Three independent bio-repeats were conducted in the experiment and each bio-repeat contains three technical replicates.

\section{Luciferase reporter assay}

The miR-3681-3p target sequences (wild-type or mutated) within the SIRT1 fragment were inserted in pmirGLO luciferase vector (Promega, Madison, WI), named as SIRT1-WT and SIRT1-Mut vectors. After co-transfection with NC mimics, miR-3681-3p mimics or miR-3681-3p mimics + circ-SIRT1-oe, cardiomyocytes were assayed with Luciferase Reporter Assay System (Promega). In addition, cardiomyocytes were co-transfected with circSIRT1-oe or NC-pcDNA3.1(+), and pGL3 vector containing SIRT1 promoter or pmirGLO vector covering SIRT1 $3^{\prime}$ UTR for luciferase assay. Three independent bio-repeats were conducted in the experiment and each biorepeat contains three technical replicates.

\section{Statistical analyses}

Data were analyzed by SPSS version 13.0 (SPSS, Chicago, IL) and displayed as the mean \pm SD. Experimental results were analyzed by one-way or twoway analysis of variance (ANOVA) or Student's $t$ test, with two-tailed $P$ value below 0.05 as a significant level.

\section{RESULTS}

\section{Circ-SIRT1 and its homologous circ-Sirt1 are low-expressed in} the $\mathrm{CH}$ model in vitro and in vivo

First, we treated human hiPSC-CMs and mouse H9c2 cells with Ang II, a known method to induce $\mathrm{CH}[29,30]$, to establish in vitro $\mathrm{CH}$ model. After treatment, the characteristics of $\mathrm{CH}$ were monitored by measuring cell surface area and sarcomere number. The results showed that the surface area of hiPSC-CMs and $\mathrm{H} 9 \mathrm{c} 2$ cells was enlarged (Fig. 1A), and the ratio of well-organized hiPSCCMs and H9c2 cells increased significantly after Ang II treatment (Supplementary Fig. 1A). So we confirmed that the $\mathrm{CH}$ cell model was successfully built by Ang II treatment.

Then, we searched circBase (http://www.circbase.org/) to find out circRNAs related to SIRT1. Consequently, 11 homo sapiens circRNAs and 1 mouse homolog circRNA were respectively associated with SIRT1 (human) and Sirt1 (mouse) gene (Supplementary Table 2). After Ang II treatment, only the expression of hsa_circ_0093884 was markedly downregulated in hiPSC-CMs (Fig. 1B). Similarly, mmu_circ_0002354 was lowexpressed in Ang II-treated H9c2 cells (Fig. 1C). Interestingly, searching circBank (http://www.circbank.cn/), we discovered that mmu_circ_0002354 happened to be a conserved mouse homolog for hsa_circ_0093884 (Fig. 1D). Thus, we speculated that hsa_circ_0093884 and its homologous mmu_circ_0002354 were involved in $\mathrm{CH}$. So we named hsa_circ_0093884 as circ-SIRT1 and mmu_circ_0002354 as circSirt 1 for further assays.

Then, to detect circ-Sirt1 level in $\mathrm{CH}$ in vivo, mouse $\mathrm{CH}$ model was built by TAC surgery. First, RT-qPCR and western blot analyses confirmed the upregulation of $\mathrm{CH}$ markers (ANF, BNP, and $\beta-M H C$ ) in TAC group (Fig. 1E, F and Supplementary Fig. 1B). Also, Ang II serum level was significantly high in the TAC group than sham group (Supplementary Fig. 1C). These data confirmed the establishment of $\mathrm{CH}$ model in vivo. Then, we verified that circ-Sirt1 expression was lower in the TAC group than that in the sham group (Fig. 1G), indicating that circ-Sirt1 participated in $\mathrm{CH}$.

Subsequently, the genomic location and the splicing pattern of circ-SIRT1 are illustrated in Fig. 1H. Nucleic acid electrophoresis uncovered that divergent primers could produce the circular isoform of SIRT1 (or Sirt1) with cDNA but not with gDNA, whereas convergent primers could amplify the linear isoform of SIRT1 (or Sirt1) from both cDNA and gDNA in hiPSC-CMs (or H9c2 cells) (Fig. 1l). After RNase R treatment, circ-SIRT1 (or circ-Sirt1) presented higher stability in contrast to linear SIRT1 (or Sirt1) (Fig. 1J).

Taken together, circ-SIRT1 and circ-Sirt1 were characterized with closed-loop structure and expressed at low levels in Ang II-treated cell models. Besides, circ-Sirt1 was low-expressed in the TAC mice model. 
A
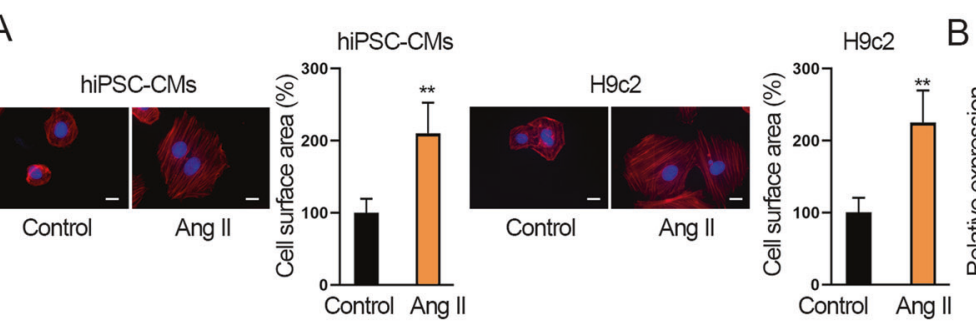

B Control 口Ang II

hiPSC-CMs
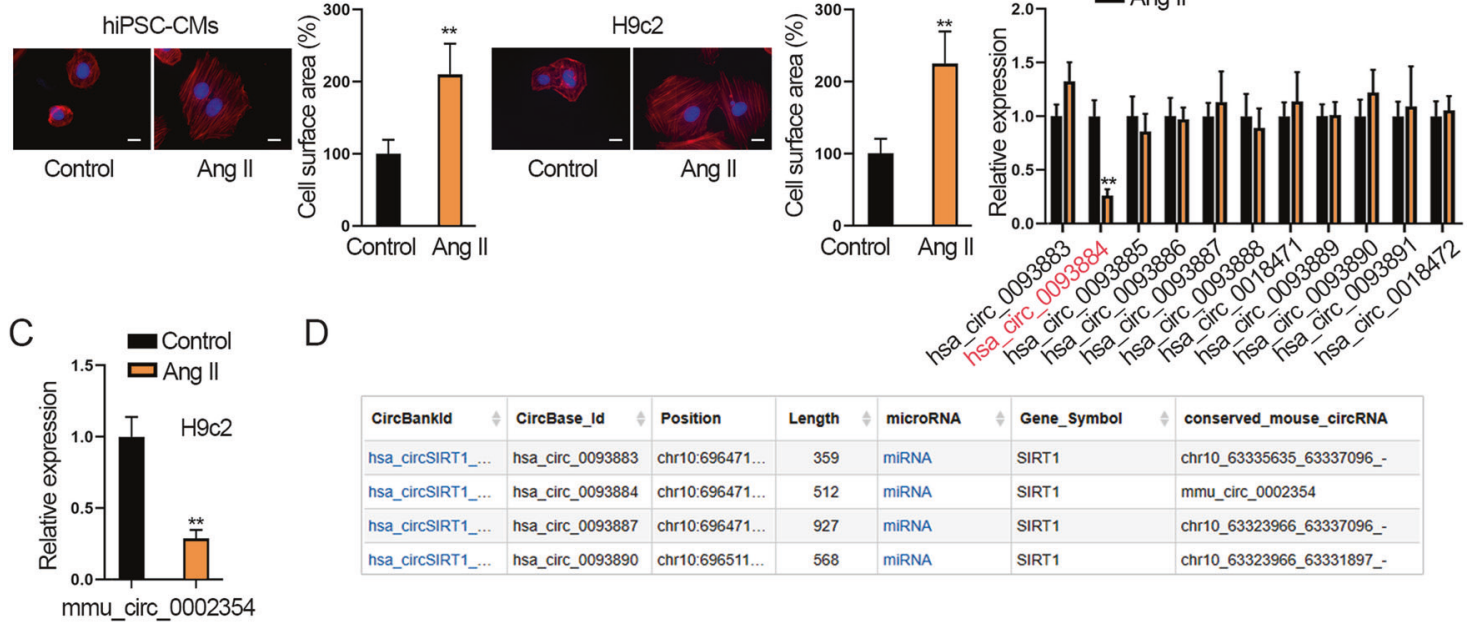

$\mathrm{D}$
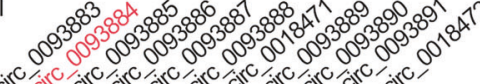

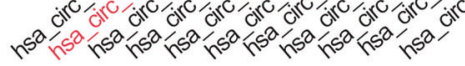

E

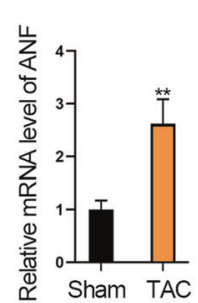

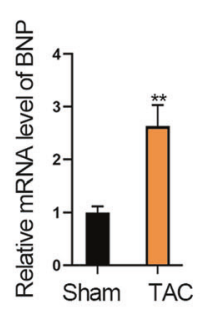

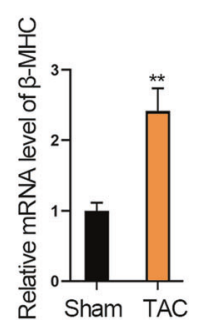

F

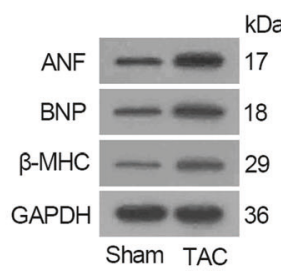

$G \stackrel{E}{E}$

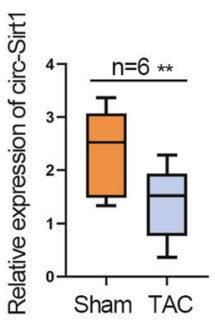

$\mathrm{H}$

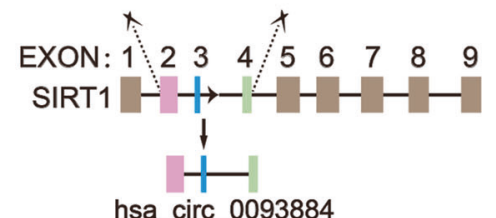

hsa_circ_0093884
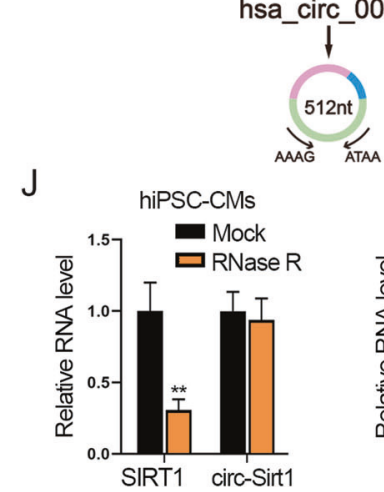

I
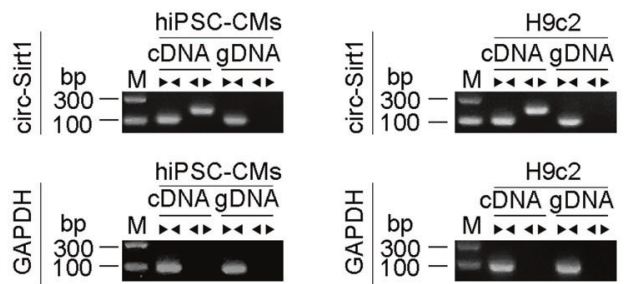
mu_circ_0002354 chr10_63323966_63337096_chr10_63323966_63331897_

\begin{tabular}{l|l|l}
568 & MIRNA & SIRT
\end{tabular}

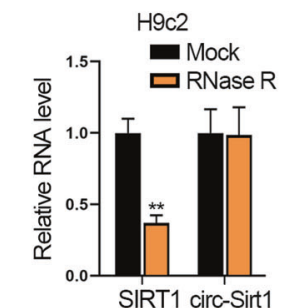

Fig. 1 Circ-SIRT1 and its homologous circ-Sirt1 are low-expressed in the CH model in vitro and in vivo. A The surface area of hiPSC-CMs and $\mathrm{H} 9 \mathrm{c} 2$ cells treated without (control) or Ang II was observed by immunofluorescence staining of $\alpha$-actinin (red). Scale bar, $10 \mu \mathrm{m}$. $N=3$. B RT-qPCR was applied to analyze the expressions of circRNAs associated with SIRT1 gene in Ang II-treated hiPSC-CMs. N=3. C RT-qPCR analysis of mmu_circ_0002354 (circ-Sirt1) expression in Ang II-treated H9c2 cells versus non-treated H9c2 cells. $N=3$. D According to the searching results in bioinformatics website circBank, mmu_circ_0002354 (circ-Sirt1) in conserved human homolog is hsa_circ_0093884 (circ-SIRT1). E, F The expression of $\mathrm{CH}$ markers (ANF, BNP, and $\beta-\mathrm{MHC}$ ) in the TAC group $(N=6)$ and sham group $(N=6)$ was examined via RT-qPCR and western blot analyses. G RT-qPCR detection of circ-Sirt1 expression in the hearts of mice subjected to TAC surgery (TAC group, $N=6)$ and sham operation (sham group, $N=6$ ). H Schematic illustration displayed the circularization of SIRT1 exon 2-4 to form hsa_circ_0093884 (circ-SIRT1). I Nucleic acid electrophoresis uncovered that divergent primers could produce the circular isoform of SIRT1 (or Sirt1) with CDNA but not with gDNA in hiPSC-CMs (or H9c2 cells). GAPDH was an internal control. $N=3$. J The abundance of circ-SIRT1/circ-Sirt1 or linear RNA SIRT1/Sirt1 analyzed via RT-qPCR after RNase R treatment in hiPSC-CMs (or H9c2 cells). $N=3{ }^{* *} P<0.01$ was assessed by Student's $t$ test.

\section{Circ-SIRT1 and homologous circ-Sirt1 inhibit $\mathrm{CH}$ and contribute to autophagy in hiPSC-MCs and H9c2 cells}

Thereafter, we explored the effect of circ-SIRT1 (or circ-Sirt1) on $\mathrm{CH}$. First, subcellular fractionation and FISH detection depicted that circ-SIRT1 and circ-Sirt1 were mainly distributed in the cytoplasm of hiPSC-CMs and H9c2 cells, respectively (Fig. 2A). As depicted by RT-qPCR data, sh-circ-SIRT1\#1/2 and sh-circ-Sirt1\#1/ 2 decreased circ-SIRT1 level in hiPSC-CMs and circ-Sirt1 level in $\mathrm{H} 9 \mathrm{C2}$ cells (Fig. 2B and Supplementary Fig. 1D). Also, FISH images depicted an efficiency circ-SIRT1 or circ-Sirt1 knockdown by sh-circ-SIRT1\#1/2 or sh-circ-Sirt1\#1/2 (Supplementary Fig. 1E). Since sh-circ-SIRT1\#1 and sh-circ-Sirt1\#1 presented better 
A
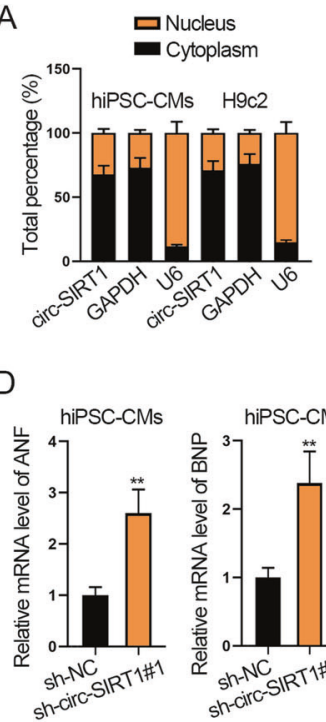

$\mathrm{H}$

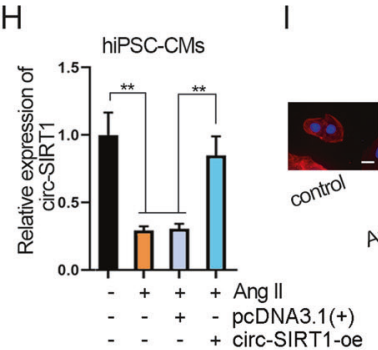

K

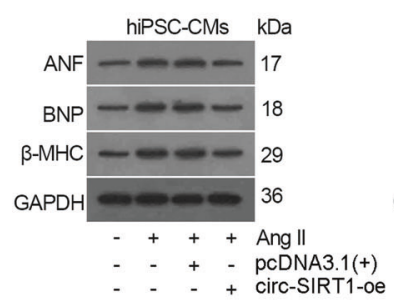

L

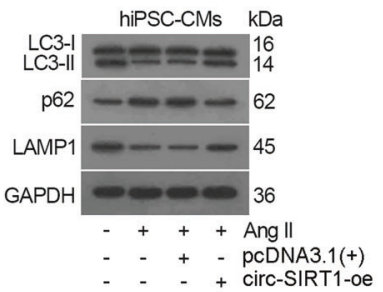

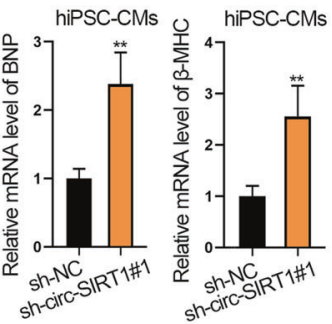
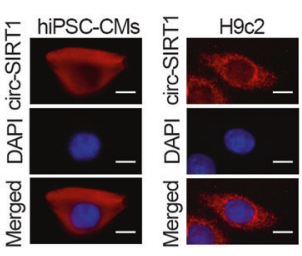

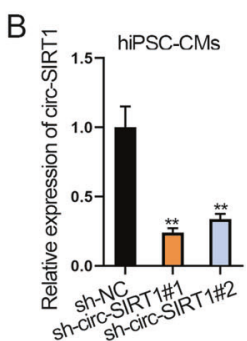

E

F
C

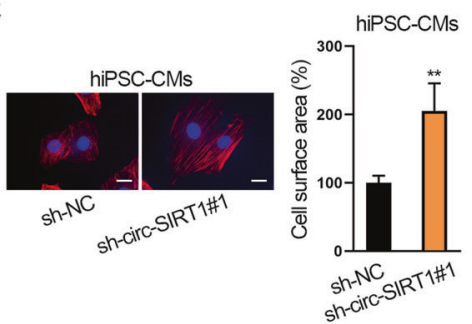

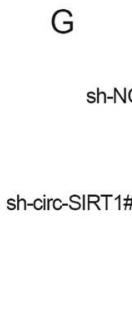

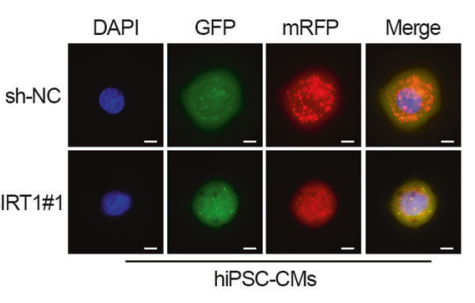

shn-NC
shoirCSTRTI

J
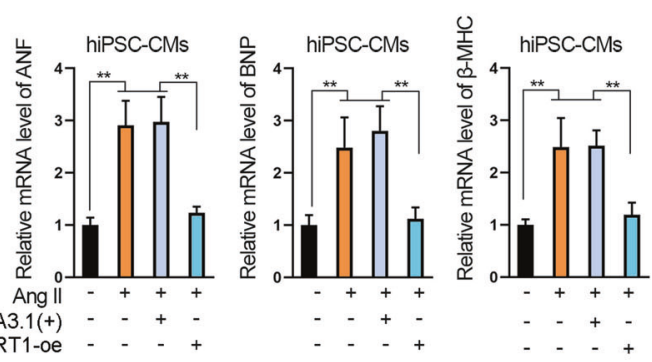

Fig. 2 Circ-SIRT1 inhibits CH and contributes to autophagy in hiPSC-MCs. A The subcellular distribution of SIRT1 (or Sirt1) was detected via subcellular fractionation and FISH assays. Scale bar, $10 \mu \mathrm{m}$. $N=3$. B RT-qPCR analysis of circ-SIRT1 knockdown by sh-circ-SIRT\#1/2 in hiPSCCMs is shown. $N=3$. C Analysis of the effects of circ-SIRT1 deficiency on cell surface area via immunofluorescence staining. Scale bar, $10 \mu m$. $N=3$. The percentage of cell surface area of the sh-circ-SIRT1\#1 group to that of the sh-NC group was evaluated. $\mathbf{D}, \mathbf{E}$ The expressions of CH markers in hiPSC-CMs transfected with sh-NC or sh-circ-SIRT1\#1 were examined via RT-qPCR and western blot analyses. $\mathbf{F}$ Western blot analysis of levels of autophagy-related proteins (LC3-II/LC3-I, p62, and LAMP1) in hiPSC-CMs transfected with sh-NC or sh-Circ-SIRT1\#1. N = 3. G The hiPSC-CMs were transfected with Ad-GFP-mRFP-LC3 for $48 \mathrm{~h}$ and sh-NC or sh-circ-SIRT1\#1. The change of both GFP fluorescence (green) and mRFP (red) fluorescence was observed by confocal microscopy. Scale bar, $10 \mu \mathrm{m} . N=3$. H Circ-SIRT1 expression in hiPSC-CMs treated without or with Ang II and transfected with pCDNA3.1 $(+)$ or pcDNA3.1 $(+)$ /circ-SIRT1 (circ-SIRT1-oe) was examined via RT-qPCR. N $=3$. I Cell surface area of hiPCS-CMs treated without or with Ang II and transfected with pcDNA3.1(+) or pcDNA3.1(+)/circ-SIRT1 (circ-SIRT1-oe) was analyzed by immunofluorescence staining. Scale bar, $10 \mu \mathrm{m} . N=3$. J, K The expression of CH markers in hiPSC-CMs of the above-mentioned groups was examined via RT-qPCR and western blot analyses. $N=3$. L, M The effect of upregulated circ-SIRT1 on autophagy influx in hiPSCCMs treated without or with Ang II and transfected with pcDNA3.1(+) or pcDNA3.1(+)/circ-SIRT1 (circ-SIRT1-oe) was analyzed via western blot analysis of autophagy-related proteins and immunofluorescence staining analysis of Ad-GFP-mRFP-LC3. Representative images of GFP dots (green), mRFP dots (red), and their merged images are shown. Scale bar, $10 \mu \mathrm{m} . N=3 .{ }^{* * *} P<0.01$ was assessed by Student's $t$ test for comparison between groups, one-way ANOVA and Tukey for multiple groups.

knockdown efficiency, we used these shRNAs for functional experiments.

Next, $\mathrm{CH}$ was determined by measuring cell surface area, sarcomere organization, and CH markers. Consequently, circ-SIRT1 silencing observably enlarged cell surface area and increased the ratio of well-organized cardiomyocytes of hiPSC-CMs (Fig. $2 \mathrm{C}$ and Supplementary Fig. 1F). In addition, knockdown of circ-SIRT1 notably elevated mRNA and protein levels of ANF, BNP, and $\beta-M H C$, indicating that circ-SIRT1 deficiency was conducive to $\mathrm{CH}$ formation (Fig. 2D, E and Supplementary Fig. 1G).

Autophagy is known to be crucial to $\mathrm{CH}$ development [16, 31], and SIRT1 (or Sirt1), the associated gene for circ-SIRT1 (or circ-Sirt1), has been proved to regulate autophagy in $\mathrm{CH}[14,15]$. Therefore, we evaluated autophagic flux in Ang II-treated hiPSC-CMs. Western blot 
showed that protein levels of LC3-II/LC3-I and LAMP1 were significantly decreased by circ-SIRT1 depletion, whereas that of p62, a polyubiquitin-binding protein known to be sequestered and degraded during autophagy [32], was markedly elevated (Fig. 2F and Supplementary Fig. 1H), suggesting the inhibitive effect of circSIRT1 downregulation on autophagy. Besides, the autophagic influx was monitored via using Ad-GFP-mRFP-LC3 expressing the GFP and mRFP fluorescent proteins for marking and tracking LC3. As the GFP fluorescent protein is sensitive to acidity, GFP fluorescence stain was quenched when lysosome-autophagosome fusion happens, only detecting red dots (mRFP). In other words, the decrease of GFP indicates the lysosome fuses with autophagosome to form autolysosome. Our results showed the numbers of GFP and mRFPpositive dots per cell were both significantly decreased after the downregulation of circ-SIRT1, so fewer red dots than yellow dots were seen in the merged images, and both autophagosomes and autolysosomes decreased under circ-SIRT1 knockdown (Fig. 2G and Supplementary Fig. 11), indicating that both autolysosome formation and LC3 expression were impeded by circ-SIRT1 knockdown in hiPSC-CMs. Thus, circ-SIRT1 knockdown restrained autophagic flux.

Meanwhile, the effect of circ-SIRT1 upregulation on $\mathrm{CH}$ and autophagy in hiPSC-CMs was analyzed. To begin with, circ-SIRT1oe significantly elevated circ-SIRT1 expression in Ang II-infused hiPSC-CMs (Fig. 2H). Unsurprisingly, circ-SIRT1 upregulation reversed the enlargement of cell surface area and the increase of well-organized cardiomyocytes ratio of hiPSC-CMs treated with Ang II (Fig. 2I and Supplementary Fig. 1J). Likewise, the upregulated circ-SIRT1 could reverse the inductive effect of Ang II on the mRNA and protein levels of $\mathrm{CH}$ markers (Fig. $2 \mathrm{~J}, \mathrm{~K}$ and
Supplementary Fig. 1K). Further, western blot analysis of autophagy-related proteins and immunofluorescence staining analysis of Ad-GFP-mRFP-LC3 were performed. The results uncovered that elevating circ-SIRT1 expression offset the effect of Ang II on decreasing LC3-II/LC3-I and LAMP1 levels and increasing p62 level (Fig. $2 \mathrm{~L}$ and Supplementary Fig. 1L). Ang II decreased the percentage of positive GFP dots and mRFP dots relative to total cells and the number of autophagosomes and autolysosomes per cell, and circ-SIRT1-oe reversed such effect (Fig. 2M and Supplementary Fig. 1M). Taken together, circ-SIRT1 inhibits $\mathrm{CH}$ formation and promotes autophagy.

Similarly, we conducted same experiments to explore the effect of circ-Sirt1 in H9c2 cells. Results showed that circ-Sirt1 knockdown increased the ratio of well-organized cells and levels of ANF, $\mathrm{BNP}, \beta-\mathrm{MHC}$, and inhibited autophagy in $\mathrm{H} 9 \mathrm{c} 2$ cells (Supplementary Fig. 2A-E). Also, Ang Il-inhibited circ-Sirt1 level was restored by circ-Sirt1 overexpression in $\mathrm{H} 9 \mathrm{c} 2$ cells (Supplementary Fig. 2F). Then, we verified that Ang II promoted $\mathrm{CH}$ development and impeded autophagy in $\mathrm{H} 9 \mathrm{c} 2$ cells, and such effects were offset by overexpressing circ-Sirt1 (Supplementary Fig. 2G-K). To sum up, circ-SIRT1 and circ-Sirt1 not only impair $\mathrm{CH}$ formation but also promote autophagy.

\section{Circ-SIRT1 suppresses CH by promoting autophagy in hiPSC- CMs}

Next, we conducted a series of rescue assays to verify whether circ-SIRT1 modulates $\mathrm{CH}$ by regulating autophagy. In the period, we used 3-methyladenine (3-MA) to interfere with autophagy. As illustrated in Fig. $3 \mathrm{~A}$ and Supplementary Fig. 3A, the addition of
A
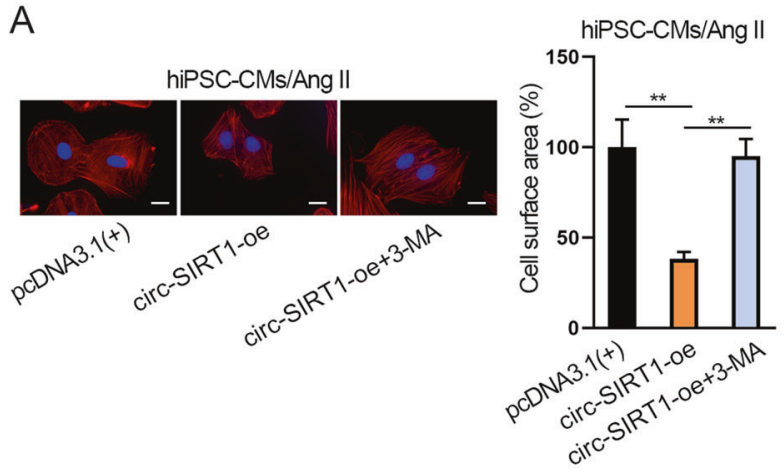

B

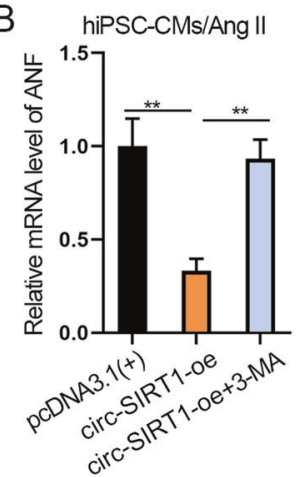

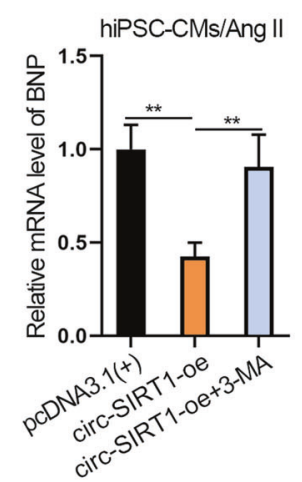
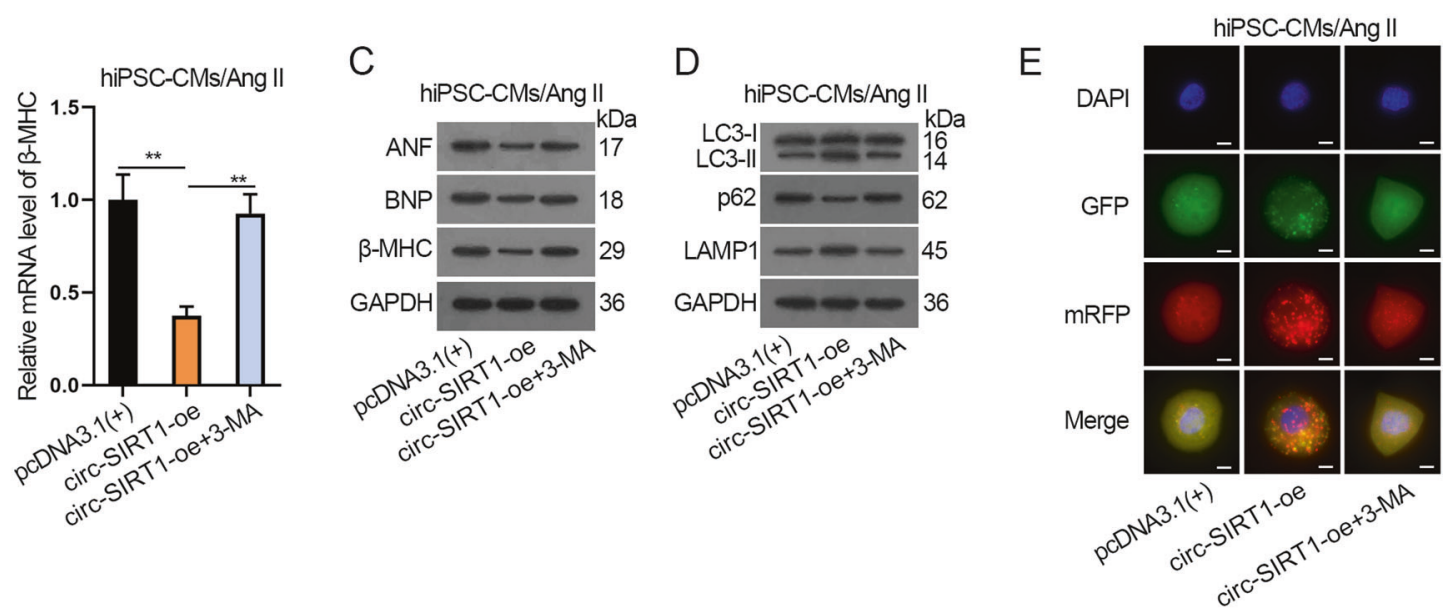

Fig. 3 Circ-SIRT1 suppresses CH by promoting autophagy in hiPSC-CMs. A Analysis of the cell surface area via immunofluorescence staining after Ang II-infused hiPSC-CMs transfected with pCDNA3.1 (+) or circ-SIRT1-oe or circ-SIRT1-oe plus treatment with 3-MA (autophagy inhibitor). Scale bar, $10 \mu \mathrm{m} . \mathrm{N}=3$. B, C The expressions of $\mathrm{CH}$ markers in Ang II-infused hiPSC-CMs of the above-mentioned groups were determined by RT-qPCR and western blot analyses. $N=3$. D, E Autophagy was measured via western blot analysis of autophagy-related proteins and immunofluorescence staining analysis of Ad-GFP-mRFP-LC3 in Ang II-infused hiPSC-CMs of the above-mentioned groups. Representative images of fluorescent GFP dots (green), mRFP dots (red), and their merged images were shown. Scale bar, $10 \mu \mathrm{m} . N=3 .{ }^{* *} P<0.01$ was assessed by one-way ANOVA and Tukey. 

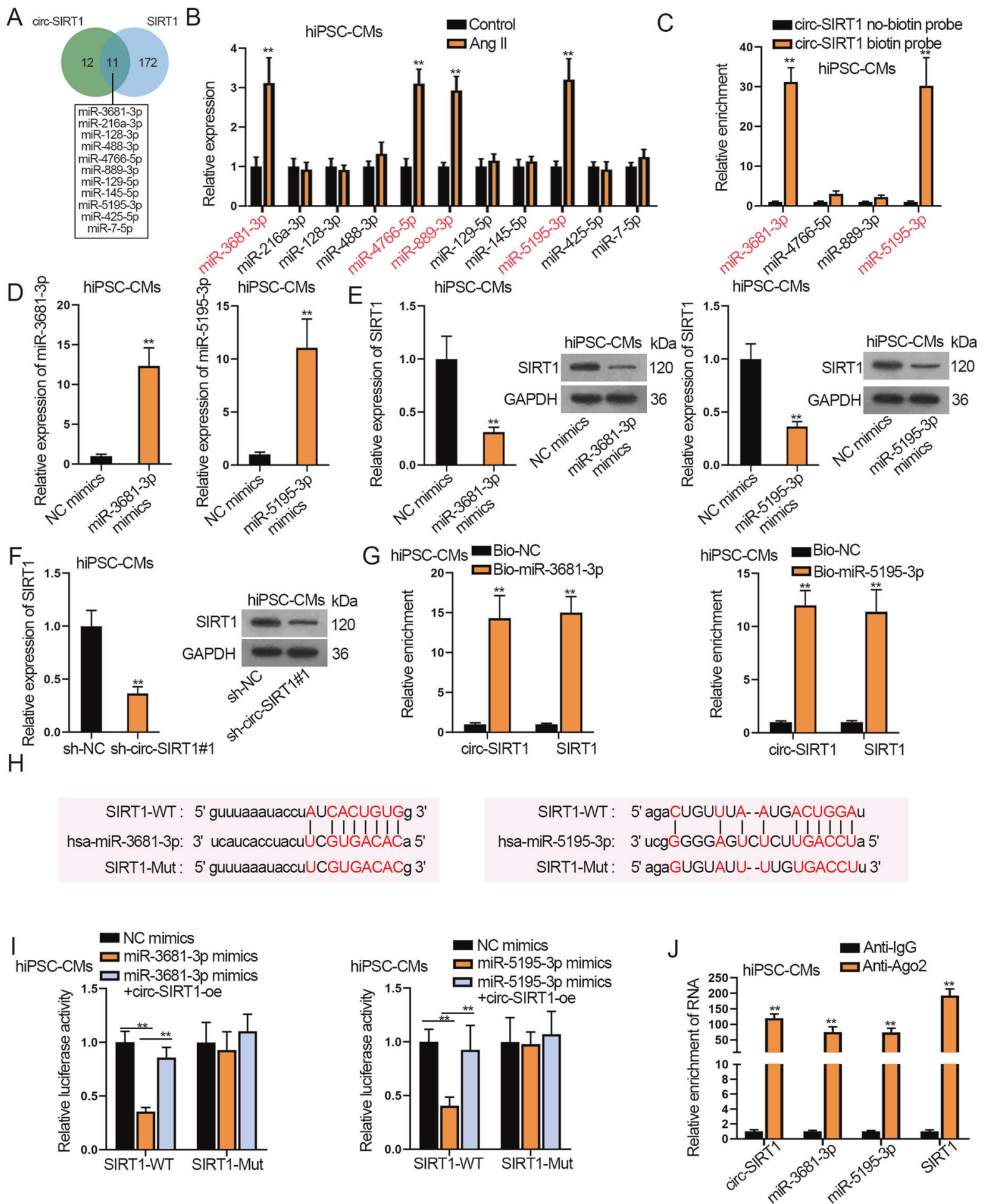

3-MA completely restored the restraining effect of circ-SIRT1 upregulation on cell surface area and the ratio of well-organized cardiomyocytes of Ang II-treated hiPSC-CMs. Unsurprisingly, the suppressive influence of circ-SIRT1 overexpression on the expression of ANF, BNP, and $\beta-M H C$ was entirely rescued by adding 3-MA to Ang II-treated hiPSC-CMs (Fig. 3B, C and Supplementary Fig. 3B). Thereby, it was suggested that circ-SIRT1 might repress $\mathrm{CH}$ by promoting autophagy. For further verification, we analyzed the levels of autophagy-related proteins. The results showed that
3-MA treatment reversed the promoting effect of circ-SIRT1 overexpression on LC3-II/LC3-I and LAMP1 protein levels and inhibitory effect of circ-SIRT1 overexpression on p62 protein in Ang II-treated hiPSC-CMs (Fig. 3D and Supplementary Fig. 3C). Also, we evaluated the extent of autophagic flux. The promoting effect of circ-SIRT1 overexpression on the numbers of GFP and mRFP-positive dots per cell were both reversed by 3-MA treatment; fewer red dots than yellow dots were seen in the merged images of circ-SIRT-oe+3-MA group compared with circ- 
Fig. 4 Circ-SIRT1 upregulates SIRT1 expression by sequestering miR-3681-3p/miR-5195-3p in hiPSC-CMs. A In total, 11 miRNAs were predicted by starBase to possess the binding capacity with circ-SIRT1 (or SIRT1). B The expression levels of candidate miRNAs in Ang II-infused hiPSC-CMs versus control hiPSC-CMs were detected via RT-qPCR. $N=3$. C RT-qPCR results of the enrichment of four miRNAs in pulldown products of circ-SIRT1 no-biotin probe or circ-SIRT1 biotin probe. Enrichment values of miRNAs were normalized to NC-biotin probe control. $N=3$. D RT-qPCR analysis of the upregulation of miR-3681-3p and miR-5195-3p in hiPSC-CMs with the transfection of miR-3681-3p mimics and miR-5195-3p mimics, respectively. $N=3$. E, F Analysis of SIRT1 expression via RT-qPCR and western blot assays in hiPSC-CMs transfected with miR-3681-3p/miR-5195-3p mimics versus NC mimics or transfected with sh-circ-SIRT1\#1 versus sh-NC. $N=3$. G RT-qPCR analysis of the enrichment of circ-SIRT1 and SIRT1 in the pulldown products of Bio-miR-3681-3p/miR-5195-3p and Bio-NC. $N=3$. Enrichment values of circSIRT1/SIRT1 were normalized to Bio-NC control. $\mathbf{H}$ The binding sites for miR-3681-3p/miR-5195-3p in SIRT1 were predicted by starBase and the sites were mutated by substitution with complementary sites (marked in red). I Luciferase activities of SIRT1-WT (with wild-type miR-3681-3p/ miR-5195-3p sites) and SIRT-Mut (with mutated miR-3681-3p/miR-5195-3p sites) were evaluated by luciferase reporter assay in hiPSC-CMs transfected with NC mimics, miR-3681-3p/miR-5195-3p mimics or miR-3681-3p/miR-5195-3p mimics+circ-SIRT1-oe. $N=3$. J The enrichment of circ-SIRT1, miR-3681-3p, miR-5195-3p, and SIRT1 in Ago2 RIP products was verified through RIP and RT-qPCR. $N=3$. ${ }^{* *} P<0.01$ was assessed by Student's $t$ test for comparison between groups, one-way ANOVA/two-way ANOVA and Tukey for multiple groups.

SIRT1-oe group in Ang II-treated hiPSC-CMs, indicating significantly decreased autolysosome formation compared with autophagosomes (Fig. 3E and Supplementary Fig. 3D). Taken together, the results above elucidated that the addition of 3-MA could completely reverse the facilitating effect of upregulated circ-SIRT1 on autophagy in Ang II-treated hiPSC-CMs.

Therefore, circ-SIRT1 impedes $\mathrm{CH}$ by promoting autophagy.

\section{Circ-SIRT1 recruits USP22 to regulate the deubiquitination and stabilization of SIRT1 in hiPSC-CMs}

Then, we tried to explain the mechanism behind the regulation of circ-SIRT1 on SIRT1 in hiPSC-CMs. First, we carried out luciferase reporter assay and observed that circ-SIRT1 upregulation caused no obvious alteration in the luciferase activity of SIRT1 promoter whereas overexpressing HIC2, a known activator for SIRT1 transcription in cardiomyocytes [33], successfully induced the luciferase activity of SIRT1 reporter (Supplementary Fig. 3E, F), but circ-SIRT1 overexpression elevated the luciferase activity of SIRT1 3'UTR (Supplementary Fig. 3G). This suggested the posttranscriptional regulation of circ-SIRT1 on SIRT1. Then, through RNA pull-down assay, we recognized that circ-SIRT1 could not bind with SIRT1 (Supplementary Fig. 3H). Next, circ-SIRT1 was unveiled to be highly enriched in Ago2 RIP precipitates (Supplementary Fig. 3l). Since Ago2 is a core protein in the RNAinduced silencing complex (RISC), the results implied that circSIRT1 existed in RISC. Combining with the former finding that circSIRT1 was mainly distributed in the cytoplasm, we speculated that circ-SIRT1 might regulate SIRT1 expression by acting as a ceRNA in hiPSC-CMs. Searching starBase (http://starbase.sysu.edu.cn/), we predicted 11 miRNAs possessing the binding capacity with both circ-SIRT1 and SIRT1 (Fig. 4A). Among them, only miR-3681-3p, miR-4766-5p, miR-889-3p, and miR-5195-3p were detected to be apparently overexpressed in Ang Il-infused hiPSC-CMs (Fig. 4B). However, only miR-3681-3p and miR-5195-3p were confirmed to be capable of binding with circ-SIRT1 in hiPSC-CMs according to the results of RNA pull-down assay (Fig. 4C). Therefore, we proceeded to examine whether circ-SIRT1 regulated SIRT1 through binding with miR-3681-3p and miR-5195-3p. First, RTqPCR analysis revealed a favorable upregulation of miR-3681-3p and miR-5195-3p in hiPSC-CMs transfected with miR-3681-3p mimics and miR-5195-3p mimics (Fig. 4D). According to RT-qPCR and western blot analyses, SIRT1 expression was proved to be decreased by the transfection of miR-3681-3p mimics, miR-5195$3 p$ mimics, or sh-circ-SIRT1\#1 (Fig. 4E, F and Supplementary Fig. 3J, $\mathrm{K})$. Later on, through RNA pull-down assay, we confirmed that miR3681-3p and miR-5195-3p could bind with circ-SIRT1 (or SIRT1) in hiPSC-CMs because we observed that circ-SIRT1 (or SIRT1) was highly abundant in Bio-miR-3681-3p and Bio-miR-5195-3p groups (Fig. 4G). In addition, Fig. 4H showed the binding sites for miR$3681-3 p / m i R-5195-3 p$ on SIRT1, and SIRT1-Mut were designed by substituting the predicted sites with complementary sequences.
Then, luciferase reporter assay indicated that upregulation of miR3681-3p or miR-5195-3p largely weakened the luciferase activity of pmirGLO-SIRT1-WT but had no marked effect on that of pmirGLOSIRT1-Mut; such effect on pmirGLO-SIRT1-WT was reversed by elevating circ-SIRT1 expression, suggesting the competitive relation between circ-SIRT1 and SIRT1 (Fig. 4I). At last, the RIP assay delineated the significant enrichment of circ-SIRT1, miR3681-3p, miR-5195-3p, and SIRT1 in the anti-Ago2 group but not in the anti-lgG group, uncovering the coexistence of these RNAs in RISC (Fig. 4J). To conclude, circ-SIRT1 elevates SIRT1 expression by competitively binding with miR-3681-3p/miR-5195-3p in hiPSCCMs.

\section{Circ-SIRT1 recruits USP22 to stabilize SIRT1 protein in hiPSC- CMs}

Interestingly, RT-qPCR and western blot analyses revealed that cotransfection of miR-3681-3p mimics plus miR-5195-3p mimics could only completely restore SIRT1 mRNA level rather than SIRT1 protein level that were reduced by circ-SIRT1 knockdown in hiPSC-CMs (Fig. 5A and Supplementary Fig. 4A). Thus, we speculated that circ-SIRT1 might regulate SIRT1 protein in other ways. CHX chase experiment revealed that circ-SIRT1 depletion accelerated SIRT1 protein degradation in hiPSC-CMs (Fig. 5B). Previous studies have clarified that SIRT1 protein stabilization was related to enzyme-mediated ubiquitination [34, 35]. Therefore, we surmised that circ-SIRT1 might regulate SIRT1 protein stability through certain proteins. The silver staining results of RNA pulldown showed the differential proteins pulled down by circ-SIRT1 biotin probe rather than NC-biotin probe (Fig. 5C). Later, these proteins were subjected to mass spectrometry analysis and results showed that USP22 was prominently enriched in the pulldown products for circ-SIRT1 in hiPSC-CMs (Supplementary Table 3). RNA pulldown and RIP assays also confirmed the strong binding capacity between circ-SIRT1 and USP22 in hiPSC-CMs (Fig. 5D). Importantly, USP22 was reported to stabilize SIRT1 by deubiquitination to promote autophagy in cancer cells and also protect against ischemia-reperfusion injury in cardiomyocytes $[36,37]$. Therefore, we speculated that circ-SIRT1 might regulate SIRT1 protein stability via USP22 and proceeded to explore the detailed mechanism. The results of RT-qPCR and western blot showed that circ-SIRT1 knockdown caused no evident changes in USP22 expression (Fig. 5E and Supplementary Fig. 4B). After that, we knocked down USP22 by sh-USP22\#1/2 and verified the knockdown efficiency (Fig. 5F and Supplementary Fig. 4C). Likewise, depleting USP22 expression in hiPSC-CMs resulted in no clear changes of circ-SIRT1 expression (Fig. 5G). Taken together, circ-SIRT1 and USP22 cannot regulate each other. Thus, we further investigated whether circ-SIRT1 recruited USP22 to stabilize SIRT1 protein. The CoIP results indicated that SIRT1 protein binds with USP22 in hiPSC-CMs (Fig. 5H). Besides, downregulation of circ-SIRT1 could inhibit the binding between 

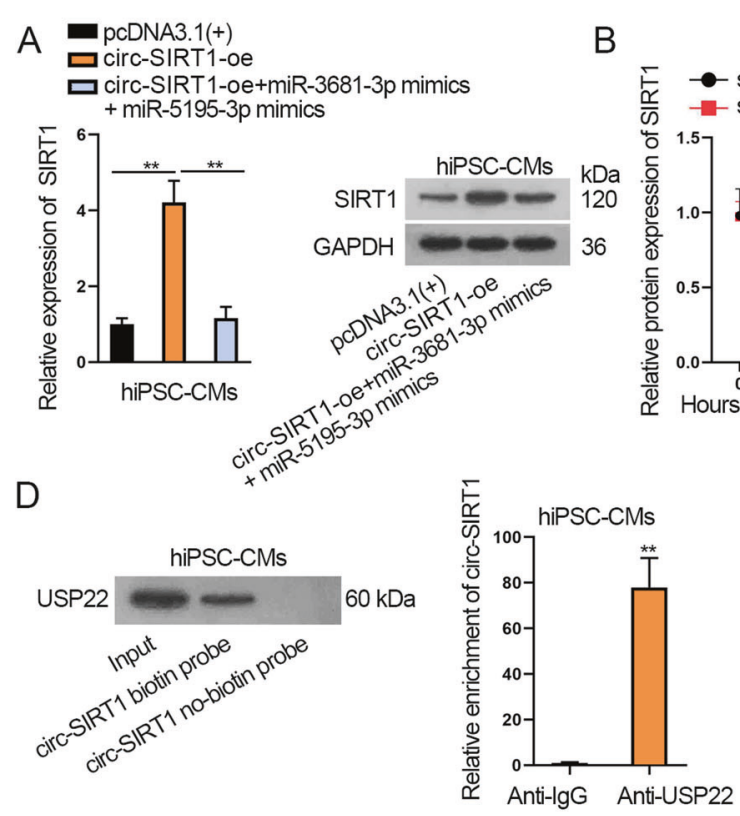

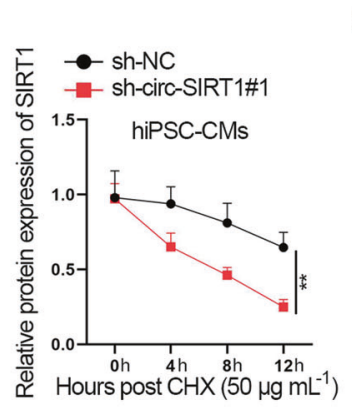

C
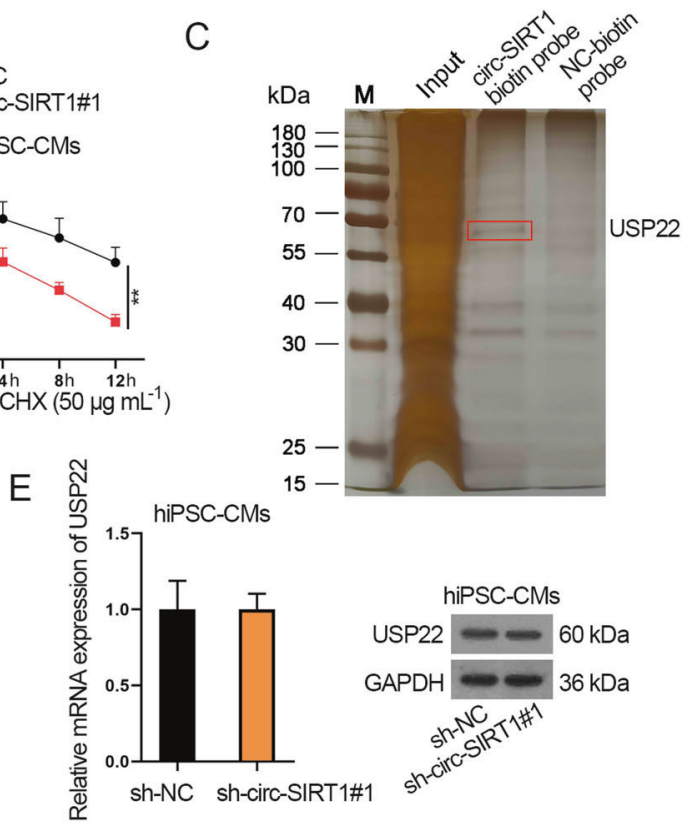
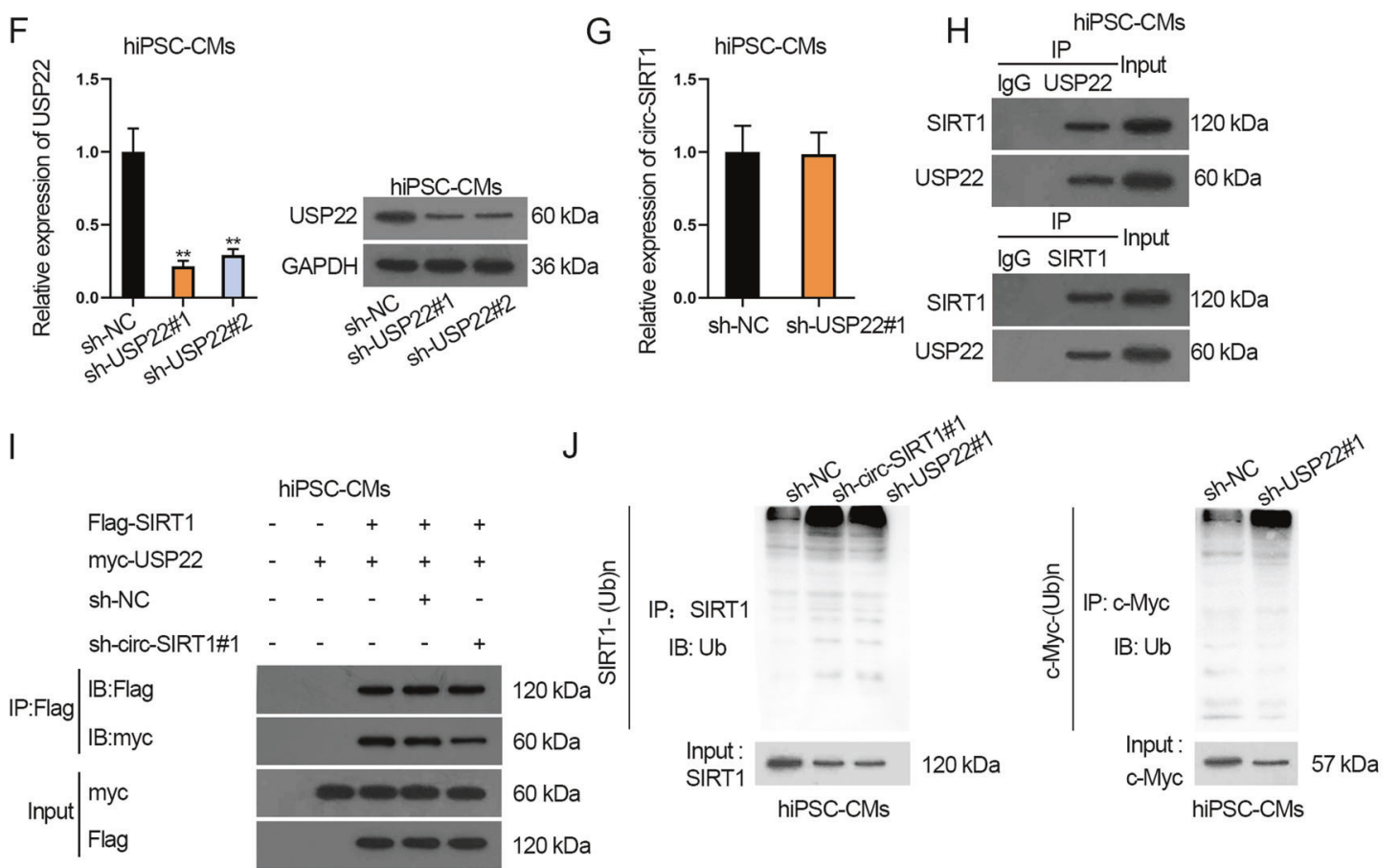

Fig. 5 Circ-SIRT1 recruits USP22 to regulate the deubiquitination and stabilization of SIRT1 in hiPSC-CMs. A SIRT1 mRNA and protein expression in hiPSC-CMs transfected with pcDNA3.1(+), circ-SIRT1-oe, or circ-SIRT1-oe+miR-3681-3p mimics+miR-5195-3p mimics were analyzed via RT-qPCR and western blot analyses. $N=3$. B SIRT1 protein stability was analyzed by western blot at $0,4,8$, and $12 \mathrm{~h}$ after $\mathrm{CHX}$ treatment in hiPSC-CMs. $N=3$. C Silver staining of differential proteins in RNA pull-down products for circ-SIRT1 biotin probe compared with NC-biotin probe. The differential bands were sent to mass spectrometry and USP22 was shown to be significantly enriched in circ-SIRT1 biotin probe group in hiPSC-CMs. $N=3$. D Western blot band of USP22 in RNA pull-down products for circ-SIRT1 biotin probe and circ-SIRT1 nobiotin probe. RT-qPCR results of circ-SIRT1 enrichment in RIP precipitates for anti-lgG and anti-USP22. $N=3$. E RT-qPCR and western blot analyzed the effect of silenced circ-SIRT1 on USP22 expression. $N=3$. F RT-qPCR and western blot analyses of the efficiency of USP22 knockdown by sh-USP22\#1/2 in hiPSC-CMs. $N=3$. G RT-qPCR analysis of the effect of silenced USP22 on circ-SIRT1 expression. $N=3$. $\mathbf{H}$ Western blot band of SIRT1 and USP22 in the ColP products for anti-lgG and anti-USP22. $N=3$. I The hiPSC-CMs were transfected with exogenous Flag-tagged SIRT1 and myc-tagged USP22 proteins and sh-NC or sh-circ-SIRT1\#1. Western blot band showed the enrichment of myc in the ColP products in anti-Flag in sh-NC and sh-circ-SIRT1\#1 groups. $N=3$. J Analysis of the effect of circ-SIRT1 depletion or USP22 deficiency on SIRT1 ubiquitination is shown, taking c-Myc as a positive control for IP. $N=3 .{ }^{* *} P<0.01$ was assessed by Student's $t$ test for comparison between groups, one-way ANOVA and Tukey for multiple groups. 


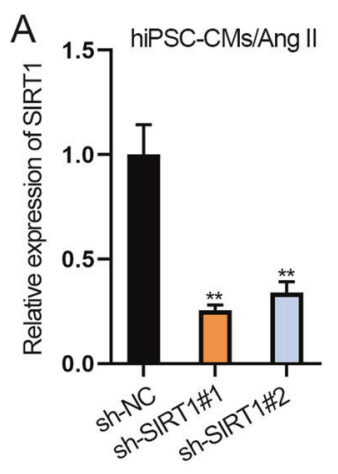

B
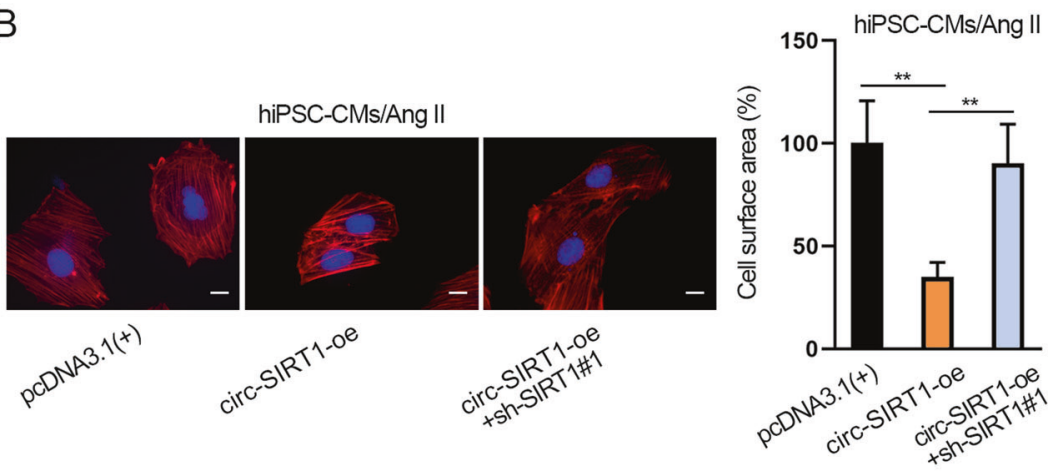

C
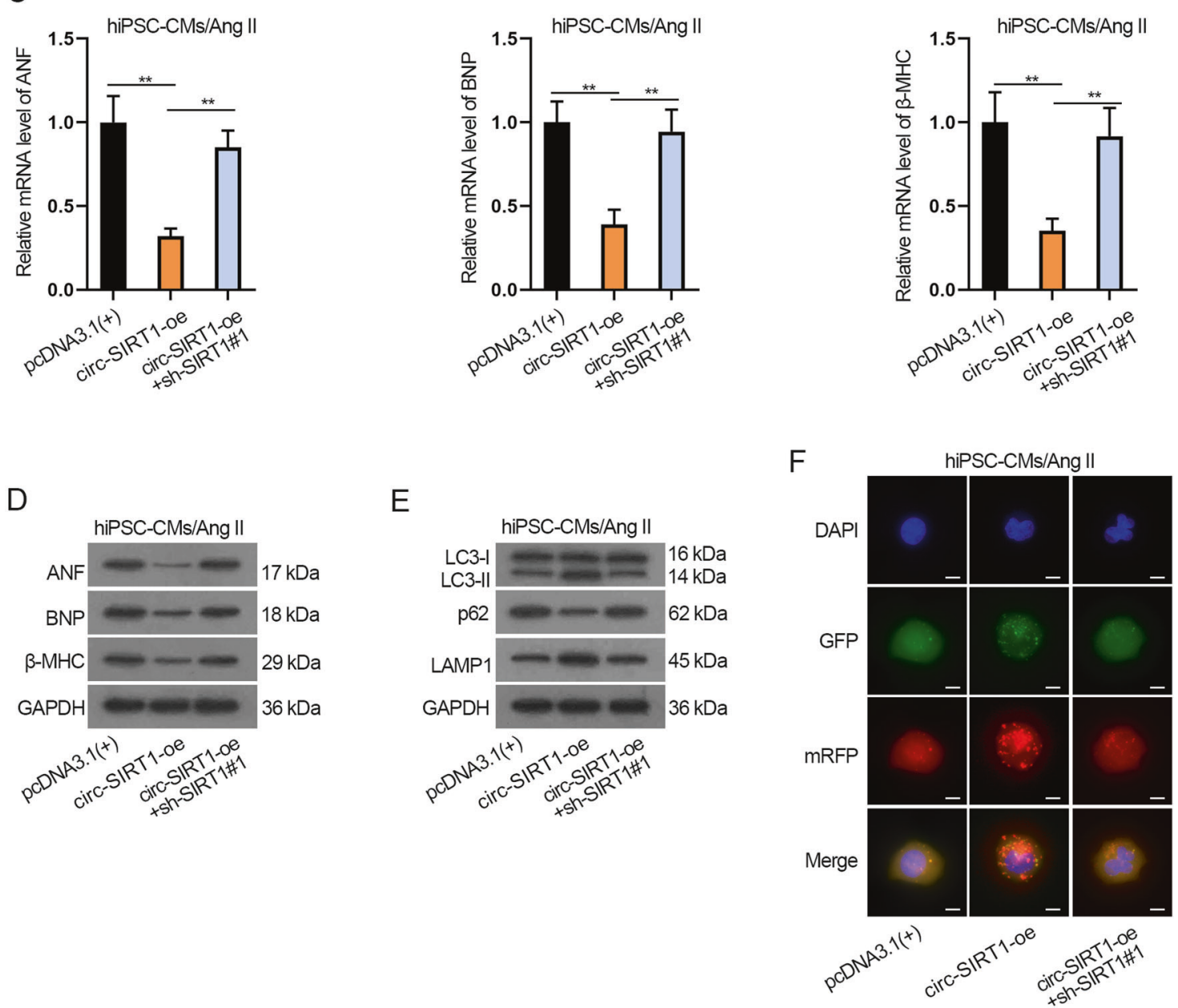

Fig. 6 Circ-SIRT1 inhibits CH via upregulating SIRT1 to enhance autophagy. A The knockdown efficiency of SIRT1 in Ang II-infused hiPSCCMs was evaluated via RT-qPCR. $N=3$. B Immunofluorescence staining analysis of the cell surface area after Ang II-infused hiPSC-CMs were transfected with pcDNDA3.1(+), circ-SIRT1-oe, or circ-SIRT1-oe+sh-circ-SIRT1\#1. Scale bar, $10 \mu \mathrm{m}$. $N=3$. C, D RT-qPCR and western blot analyses of the expression of CH markers after Ang Il-infused hiPSC-CMs were transfected with pcDNDA3.1(+), circ-SIRT1-oe, or circ-SIRT1-oe + sh-circ-SIRT1\#1. $N=3$. E, F Autophagy in Ang Il-infused hiPSC-CMs of the above-mentioned groups was measured by western blot analysis of autophagy-related proteins and immunofluorescence staining analysis of Ad-GFP-mRFP-LC3. Representative images of fluorescent GFP dots (green), mRFP dots (red), and their merged images are shown. Scale bar, $10 \mu \mathrm{m} . N=3$. $^{* *} P<0.01$ was assessed by one-way ANOVA and Tukey.

SIRT1 and USP22 (Fig. 5I). More importantly, either circ-SIRT1 depletion or USP22 knockdown could promote the ubiquitination of SIRT1 (Fig. 5J), indicating that circ-SIRT1 facilitated the USP22mediated deubiquitination of SIRT1, promoting the stability of SIRT1 protein.

All in all, circ-SIRT1 stabilizes SIRT1 protein via recruiting USP22 to facilitate the deubiquitination of SIRT1 in hiPSC-CMs.

\section{Circ-SIRT1 inhibits CH via upregulating SIRT1 to enhance autophagy}

At last, examined the influence of circ-SIRT1/SIRT1 axis on $\mathrm{CH}$ and autophagy by rescue experiments. First, the knockdown efficiency of SIRT1 was evaluated via RT-qPCR and confirmed that sh-SIRT1\#1 presented a better knockdown efficiency in Ang II-treated hiPSCMCs (Fig. 6A). Thereby, we adopted sh-SIRT1\#1 for follow-up 
experiments. As shown in Fig. $6 \mathrm{~B}$ and Supplementary Fig. 4D, SIRT1 knockdown reversed the suppressive impact of circ-SIRT1 overexpression on cell surface area and the ratio of well-organized cardiomyocytes of Ang II-treated hiPSC-CMs. Likewise, the suppressive influence of circ-SIRT1 upregulation on the expression of ANF, BNP, and $\beta-M H C$ was reversed by SIRT1 deficiency in Ang II-infused hiPSC-CMs (Fig. 6C, D and Supplementary Fig. 4E). These data indicated that SIRT1 knockdown countervails the inhibitory effect of circ-SIRT1 on $\mathrm{CH}$. In addition, SIRT1 depletion reverses the promoting effect of circ-SIRT1 overexpression on autophagy in Ang Il-treated hiPSC-CMs (Fig. 6E, F and Supplementary Fig. 4F, G). In a word, circ-SIRT1 inhibits $\mathrm{CH}$ and promotes autophagy via modulating SIRT1 in Ang II-treated $\mathrm{CH}$ cell model.

Besides, we established another $\mathrm{CH}$ model in vitro by ISOtreatment to verify the function of circ-SIRT1/SIRT1 on $\mathrm{CH}$ via autophagy pathway. The results demonstrated that circ-SIRT1 overexpression reversed the improvement of $\mathrm{CH}$ and suppression of autophagy in ISO-treated hiPSC-CMs, and such effect was abrogated by sh-SIRT1\#1 or 3-MA (Supplementary Fig. 5A-E).

In addition to in vitro model, we explored the effect of circ-Sirt1 on $\mathrm{CH}$ in Ang II-induced in vivo mice model. According to RT-qPCR and western blot analyses, Ang II infusion overtly induced the mRNA and protein levels of ANF, BNP, and $\beta-M H C$, and such effect was abolished by circ-Sirt1 overexpression (Supplementary Fig. 6A). Moreover, circ-Sirt1 and Sirt1 were reduced by Ang II infusion in mouse hearts and overexpression of circ-Sirt1 reversed such effect (Supplementary Fig. 6B). Thus, circ-Sirt1 inhibits $\mathrm{CH}$ by upregulating Sirt1 in vivo.

\section{DISCUSSION}

It is known that persistent cardiac hypertrophic growth is tightly linked to adverse consequences that may ultimately induce heart failure and also sudden death [38, 39]. Thus, researches are demanded for deeper understanding of the mechanism of its pathology. So far, numerous researches have uncovered a wide range of genes regulating $\mathrm{CH}$, such as HSF1 and EndoA1 $[40,41]$. Interestingly, existing evidence has clarified the negative relation of autophagy to $\mathrm{CH}$, that is, promoting autophagy may attenuate $\mathrm{CH}[16,31]$. SIRT1 is one of the histone deacetylases (HDACs) that bind to protein lysine residues to trigger de-acetylation [42]. Recently, SIRT1 has been manifested to elicit promoting effect on autophagy by de-acetylation of certain genes, such as Beclin 1 and ATG9A [14, 15]. Importantly, as previously reported, SIRT1 contributed to the inhibition of $\mathrm{CH}$ and SIRT1 was regulated through multiple mechanisms in $\mathrm{CH}$, such as being inhibited by miR-122 or promoted by SP1 and FGF21 [18-20]. CircRNAs are recently discovered to play a part in $\mathrm{CH}$ [43]. Although regulation of SIRT1 by circRNAs has been elucidated in some cancers and diseases [25, 27, 44], our study was the first to uncover the circRNA-mediated regulation of SIRT1 in $\mathrm{CH}$. In this study, through bioinformatics and biological assays, we searched out several circRNAs associated with SIRT1. We then proved that circ-SIRT1 and its homologous mouse circ-Sirt1 were low-expressed in Ang IIor ISO-induced $\mathrm{CH}$ cell model in vitro and in Ang II infusioninduced $\mathrm{CH}$ mice model in vivo, supporting the link between circSIRT1 (and circ-Sirt1) and CH. Also, functional assay suggested that circ-SIRT1 and circ-Sirt1 could inhibit $\mathrm{CH}$ and improve autophagy in human and mouse cardiomyocytes. Furthermore, by using 3MA, the autophagy inhibitor, designed rescue assays and proved that inhibiting autophagy can block the inhibitory effect of circSIRT1 on $\mathrm{CH}$, suggesting that circ-SIRT1 regulated $\mathrm{CH}$ via autophagy pathway. Although circ-SIRT1 was demonstrated to regulated SIRT1 in vascular smooth muscle cells [45], our study first identified that circ-SIRT1 was involved as a negative regulator for $\mathrm{CH}$.

Previous investigations have indicated that circRNAs as ceRNAs were able to regulate multiple cellular behaviors via competing with mRNAs for miRNAs $[46,47]$. In this research, we showed that circ-SIRT1 was abundant in the cytoplasm and regulated the luciferase activity of SIRT1 3'UTR but not SIRT1 promoter, indicating circ-SIRT1 might post-transcriptionally regulate SIRT1 expression by acting as a ceRNA of miRNAs in hiPSC-CMs. Expectedly, we predicted and confirmed that miR-3681-3p and miR-5195-3p bound with circ-SIRT1 (or SIRT1) in hiPSC-CMs, and circ-SIRT1 could regulate SIRT1 expression via competitively binding with miR-3681-3p/miR-5195-3p. Previously, miR-3681-3p was documented to participate in acute and chronic hepatitis $B$ virus infection, and miR-5195-3p were supported to suppress the activity of several cancer cells [48, 49], however, our data first established the link between miR-3681-3p/miR-5195-3p with $\mathrm{CH}$. Interestingly, rescue assays confirmed that upregulation of miR3681-3p and miR-5195-3p could completely reverse the effects of circ-SIRT1 upregulation on SIRT1 mRNA expression but partially on SIRT1 protein expression, suggesting the existence of another pathway in the regulation of SIRT1 protein expression.

By RNA-pulldown and mass spectrometry, we identified that USP22 bound with circ-SIRT1 in cardiomyocytes. USP22 is a deubiquitinating enzyme (DUB) as one member of the ubiquitinspecific protease (USP) superfamily [50]. It has been manifested that SIRT1 protein stability is associated with enzyme-mediated ubiquitination [34, 35]. More importantly, as formally reported, USP22 promotes Sirt1 deubiquitination and stabilization to restrains cell apoptosis [34]. Herein, our study first demonstrated that USP22 also regulated the deubiquitination of SIRT1 in $\mathrm{CH}$ and we first discovered that decreased expression of circ-SIRT1 restrained the binding of USP22 with SIRT1, suggesting that circSIRT1 promotes the deubiquitination and stabilization of SIRT1 by recruiting USP22 in hiPSC-CMs. Finally, rescue assays delineated that knockdown of SIRT1 could rescue the effect of circ-SIRT1 upregulation on autophagy and $\mathrm{CH}$, confirming the suppressive function of circ-SIRT1/SIRT1 axis in $\mathrm{CH}$.

In conclusion, our study first demonstrated that circ-SIRT1 inhibits $\mathrm{CH}$ via upregulating and stabilizing SIRT1 to promote autophagy. This finding provides evidence of the vital function of circ-SIRT1 on regulating autophagy and $\mathrm{CH}$, and sheds novel light on the improvement of $\mathrm{CH}$ treatment.

\section{DATA AVAILABILITY}

The data have been provided.

\section{REFERENCES}

1. Oka T, Akazawa H, Naito AT, Komuro I. Angiogenesis and cardiac hypertrophy: maintenance of cardiac function and causative roles in heart failure. Circulation Res. 2014;114:565-71

2. Bernardo BC, Weeks KL, Pretorius L, McMullen JR. et al. Molecular distinction between physiological and pathological cardiac hypertrophy: experimental findings and therapeutic strategies. Pharmacol Therapeutics. 2010;128:191-227. https://doi.org/10.1126/scisignal.aaf5967.

3. Dadson K, Hauck L, Billia F. Molecular mechanisms in cardiomyopathy. Clin Sci. 2017;131:1375-92.

4. Hunter JJ, Chien KR. Signaling pathways for cardiac hypertrophy and failure. N Engl J Med. 1999;341:1276-83.

5. Frangogiannis NG. Pathophysiology of myocardial infarction. Comprehensive Physiol. 2015;5:1841-75.

6. Driver JA, Djousse L, Logroscino G, Gaziano JM, Kurth T. Incidence of cardiovascular disease and cancer in advanced age: prospective cohort study. BMJ (Clin Res Ed). 2008;337:a2467.

7. Dolinsky VW, Morton JS, Oka T, Robillard-Frayne I, Bagdan M, Lopaschuk GD, et al. Calorie restriction prevents hypertension and cardiac hypertrophy in the spontaneously hypertensive rat. Hypertension. 2010;56:412-21.

8. Colman RJ, Anderson RM, Johnson SC, Kastman EK, Kosmatka KJ, Beasley TM, et al. Caloric restriction delays disease onset and mortality in rhesus monkeys. Science. 2009;325:201-4.

9. Fontana L, Klein S. Aging, adiposity, and calorie restriction. J Am Med Assoc. 2007;297:986-94. 
10. Lyon RC, Zanella F, Omens JH, Sheikh F. Mechanotransduction in cardiac hypertrophy and failure. Circulation Res. 2015;116:1462-76.

11. Tang X, Chen XF, Wang NY, Wang XM, Liang ST, Zheng W, et al. SIRT2 acts as a cardioprotective deacetylase in pathological cardiac hypertrophy. Circulation. 2017; 136:2051-67.

12. Pashler AL, Towler BP, Jones $\mathrm{Cl}$, Newbury SF. The roles of the exoribonucleases DIS3L2 and XRN1 in human disease. Biochemical Soc Trans. 2016;44:1377-84.

13. Esteller M. Non-coding RNAs in human disease. Nat Rev Genet. 2011;12:861-74.

14. Sun T, Li X, Zhang P, Chen WD, Zhang HL, Li DD, et al. Acetylation of Beclin 1 inhibits autophagosome maturation and promotes tumour growth. Nat Commun. 2015;6:7215.

15. Pang J, Xiong $\mathrm{H}, \mathrm{Ou} \mathrm{Y}, \mathrm{Yang} \mathrm{H}, \mathrm{Xu} \mathrm{Y}, \mathrm{Chen} \mathrm{S}$, et al. SIRT1 protects cochlear hair cell and delays age-related hearing loss via autophagy. Neurobiol Aging. 2019;80:127-37.

16. Simonson B, Subramanya V, Chan MC, Zhang A, Franchino H, Ottaviano F. et al. DDiT4L promotes autophagy and inhibits pathological cardiac hypertrophy in response to stress. Sci Signaling. 2017;10:eaaf5967 https://doi.org/10.1126/ scisignal.aaf5967.

17. Liu BY, Li L, Liu GL, Ding W, Chang WG, Xu T, et al. Baicalein attenuates cardiac hypertrophy in mice via suppressing oxidative stress and activating autophagy in cardiomyocytes. Acta Pharmacologica Sin. 2021;42:701-14.

18. Huang ZP, Chen J, Seok HY, Zhang Z, Kataoka M, Hu X, et al. MicroRNA-22 regulates cardiac hypertrophy and remodeling in response to stress. Circulation Res. 2013;112:1234-43.

19. Dong ZX, Wan L, Wang RJ, Shi YQ, Liu GZ, Zheng SJ, et al. (-)-Epicatechin suppresses angiotensin II-induced cardiac hypertrophy via the activation of the SP1/ SIRT1 signaling pathway. Cell Physiol Biochem: Int J Exp Cell Physiol Biochem, Pharmacol. 2017;41:2004-15.

20. Li S, Zhu Z, Xue M, Yi X, Liang J, Niu C, et al. Fibroblast growth factor 21 protects the heart from angiotensin II-induced cardiac hypertrophy and dysfunction via SIRT1. Biochimica et Biophysica Acta Mol Basis Dis. 2019;1865:1241-52.

21. ENCODE Project Consortium. An integrated encyclopedia of DNA elements in the human genome. Nature. 2012;489:57-74.

22. Qureshi IA, Mehler MF. Emerging roles of non-coding RNAs in brain evolution, development, plasticity and disease. Nat Rev Neurosci. 2012;13:528-41.

23. Hansen TB, Jensen TI, Clausen BH, Bramsen JB, Finsen B, Damgaard CK, et al. Natural RNA circles function as efficient microRNA sponges. Nature. 2013;495:384-8.

24. Memczak S, Jens M, Elefsinioti A, Torti F, Krueger J, Rybak A, et al. Circular RNAs are a large class of animal RNAs with regulatory potency. Nature. 2013;495:333-8.

25. Yao Y, Hua Q, Zhou Y, Shen H. CircRNA has_circ_0001946 promotes cell growth in lung adenocarcinoma by regulating miR-135a-5p/SIRT1 axis and activating Wnt/ beta-catenin signaling pathway. Biomedicine Pharmacother $=$ Biomedecine Pharmacotherapie. 2019;111:1367-75.

26. Lei B, Huang Y, Zhou Z, Zhao Y, Thapa AJ, Li W, et al. Circular RNA hsa_circ_0076248 promotes oncogenesis of glioma by sponging miR-181a to modulate SIRT1 expression. J Cell Biochem. 2019;120:6698-708.

27. Kong $P, Y u Y$, Wang $L$, Dou $Y Q$, Zhang $X H$, Cui $Y$, et al. circ-Sirt1 controls NFkappaB activation via sequence-specific interaction and enhancement of SIRT1 expression by binding to miR-132/212 in vascular smooth muscle cells. Nucleic Acids Res. 2019:47:3580-93.

28. Kabeya Y, Mizushima N, Ueno T, Yamamoto A, Kirisako T, Noda T, et al. LC3, a mammalian homologue of yeast Apg8p, is localized in autophagosome membranes after processing. EMBO J. 2000;19:5720-8.

29. Wang L, Ye N, Lian X, Peng F, Zhang H, Gong H. MiR-208a-3p aggravates autophagy through the PDCD4-ATG5 pathway in Ang Il-induced H9c2 cardiomyoblasts. Biomedicine Pharmacother = Biomedecine Pharmacotherapie. 2018;98:1-8.

30. Luo YX, Tang X, An XZ, Xie XM, Chen XF, Zhao X, et al. SIRT4 accelerates Ang IIinduced pathological cardiac hypertrophy by inhibiting manganese superoxide dismutase activity. Eur Heart J. 2017;38:1389-98.

31. Viereck J, Kumarswamy R, Foinquinos A, Xiao K, Avramopoulos $P$, Kunz $M$, et al. Long noncoding RNA chast promotes cardiac remodeling. Sci Transl Med. 2016;8:326ra22.

32. Hariharan N, Zhai $P$, Sadoshima J. Oxidative stress stimulates autophagic flux during ischemia/reperfusion. Antioxid Redox Signal. 2011;14:2179-90.

33. Song JY, Lee SH, Kim MK, Jeon BN, Cho SY, Lee SH, et al. HIC2, a new transcription activator of SIRT1. FEBS Lett. 2019;593:1763-76.

34. Lin Z, Yang $H$, Kong Q, Li J, Lee SM, Gao B, et al. USP22 antagonizes p53 transcriptional activation by deubiquitinating Sirt1 to suppress cell apoptosis and is required for mouse embryonic development. Mol Cell. 2012;46:484-94.

35. Ren X, Chen N, Chen Y, Liu W, Hu Y. TRB3 stimulates SIRT1 degradation and induces insulin resistance by lipotoxicity via COP1. Exp Cell Res. 2019;382:111428.

36. Xiong $\mathrm{H}, \mathrm{Ni} \mathrm{Z}$, He J, Jiang $\mathrm{S}$, Li X, He J, et al. LnCRNA HULC triggers autophagy via stabilizing Sirt1 and attenuates the chemosensitivity of HCC cells. Oncogene. 2017;36:3528-40.
37. Ma S, Sun L, Wu W, Wu J, Sun Z, Ren J. USP22 protects against myocardial ischemia-reperfusion injury via the SIRT1-p53/SLC7A11-dependent inhibition of ferroptosis-induced cardiomyocyte death. Front Physiol. 2020;11:551318.

38. Berk BC, Fujiwara K, Lehoux S. ECM remodeling in hypertensive heart disease. J Clin Investig. 2007;117:568-75.

39. Hill JA, Olson EN. Cardiac plasticity. N. Engl J Med. 2008;358:1370-80.

40. Yuan L, Qiu L, Ye Y, Wu J, Wang S, Wang X, et al. Heat-shock transcription factor 1 is critically involved in the ischaemia-induced cardiac hypertrophy via JAK2/ STAT3 pathway. J Cell Mol Med. 2018;22:4292-303.

41. Liu Y, Shen $H J$, Wang $X Q$, Liu $H Q$, Zheng LY, Luo JD. EndophilinA2 protects against angiotensin II-induced cardiac hypertrophy by inhibiting angiotensin II type 1 receptor trafficking in neonatal rat cardiomyocytes. J Cell Biochem. 2018;119:8290-303.

42. Roth $M$, Chen WY. Sorting out functions of sirtuins in cancer. Oncogene. 2014;33:1609-20.

43. Lavenniah A, Luu TDA, Li YP, Lim TB, Jiang J, Ackers-Johnson M, et al. Engineered circular RNA sponges act as miRNA inhibitors to attenuate pressure overload-induced cardiac hypertrophy. Mol Ther: J Am Soc Gene Ther. 2020;28:1506-17.

44. Song H, Bian ZX, Li HY, Zhang Y, Ma J, Chen SH, et al. Characterization of hsa_circ_0000594 as a new biomarker and therapeutic target for hepatoblastoma. Eur Rev Med Pharmacol Sci. 2019;23:8274-86.

45. Kong $P, Y u$ Y, Wang L, Dou YQ, Zhang XH, Cui Y, et al. circ-Sirt1 controls NF-KB activation via sequence-specific interaction and enhancement of SIRT1 expression by binding to miR-132/212 in vascular smooth muscle cells. Nucleic Acids Res. 2019;47:35-93.

46. Sun M, Zhao W, Chen Z, Li M, Li S, Wu B, et al. Circ_0058063 regulates CDK6 to promote bladder cancer progression by sponging miR-145-5p. J Cell Physiol. 2019;234:4812-24.

47. Chen T, Yang Z, Liu C, Wang L, Yang J, Chen L, et al. Circ_0078767 suppresses non-small-cell lung cancer by protecting RASSF1A expression via sponging miR330-3p. Cell Prolif. 2019;52:e12548.

48. Li Y, Jiang A. ST8SIA6-AS1 promotes hepatocellular carcinoma by absorbing miR5195-3p to regulate HOXB6. Cancer Biol Ther. 2020;21:647-55.

49. Jiang Z, Zhang Y, Cao R, Li L, Zhong K, Chen Q, et al. MiR-5195-3p inhibits proliferation and invasion of human bladder cancer cells by directly targeting oncogene KLF5. Oncol Res. 2017;25:1081.

50. Roedig J, Kowald L, Juretschke T, Karlowitz R, Ahangarian Abhari B, Roedig H, et al. USP22 controls necroptosis by regulating receptor-interacting protein kinase 3 ubiquitination. EMBO Rep. 2021;22:e50163-e.

\section{ACKNOWLEDGEMENTS}

We appreciate the supports of the First Hospital of China Medical University.

\section{AUTHOR CONTRIBUTIONS}

Weichen Wang: data analysis; LW: investigation and preparation; MY: data record; CW: study design, experiments, and data curation; RL: manuscript review; Weiwei Wang: experiments and interpretation; YL: drafted the manuscript.

\section{COMPETING INTERESTS}

The authors declare no competing interests.

\section{ETHICS STATEMENT}

The animal-related protocol was approved by the Animal Care and Use Committee of the First Hospital of China Medical University.

\section{ADDITIONAL INFORMATION}

Supplementary information The online version contains supplementary material available at https://doi.org/10.1038/s41419-021-04059-y.

Correspondence and requests for materials should be addressed to Yuze Li.

Reprints and permission information is available at http://www.nature.com/ reprints

Publisher's note Springer Nature remains neutral with regard to jurisdictional claims in published maps and institutional affiliations. 
Open Access This article is licensed under a Creative Commons At c) Atribution 4.0 International License, which permits use, sharing,
adaptation, distribution and reproduction in any medium or format, as long as you give appropriate credit to the original author(s) and the source, provide a link to the Creative Commons license, and indicate if changes were made. The images or other third party material in this article are included in the article's Creative Commons license, unless indicated otherwise in a credit line to the material. If material is not included in the article's Creative Commons license and your intended use is not permitted by statutory regulation or exceeds the permitted use, you will need to obtain permission directly from the copyright holder. To view a copy of this license, visit http://creativecommons. org/licenses/by/4.0/.

(c) The Author(s) 2021 